\title{
Diversity of mosquitoes and the aquatic insects associated with their oviposition sites along the Pacific coast of Mexico
}

\author{
J Guillermo Bond ${ }^{1}$, Mauricio Casas-Martínez ${ }^{1}$, Humberto Quiroz-Martínez², Rodolfo Novelo-Gutiérrez³, \\ Carlos F Marina', Armando Ulloa', Arnoldo Orozco-Bonilla', Miguel Muñoz ${ }^{1}$ and Trevor Williams ${ }^{{ }^{*}}$
}

\begin{abstract}
Background: The abundance, richness and diversity of mosquitoes and aquatic insects associated with their oviposition sites were surveyed along eight states of the Pacific coast of Mexico. Diversity was estimated using the Shannon index $\left(\mathrm{H}^{\prime}\right)$, similarity measures and cluster analysis.

Methods: Oviposition sites were sampled during 2-3 months per year, over a three year period. Field collected larvae and pupae were reared and identified to species following adult emergence. Aquatic insects present at oviposition sites were also collected, counted and identified to species or genus.

Results: In total, 15 genera and 74 species of mosquitoes were identified: Anopheles pseudopunctipennis, An. albimanus and Aedes aegypti were the most abundant and widely-distributed species, representing $47 \%$ of total mosquito individuals sampled. New species records for certain states are reported. Anopheline diversity was lowest in Sinaloa state $\left(H^{\prime}=0.54\right)$ and highest in Chiapas $\left(H^{\prime}=1.61\right)$ and Michoacán $\left(H^{\prime}=1.56\right)$, whereas culicid diversity was lowest in Michoacán $\left(H^{\prime}=1.93\right)$, Colima $\left(H^{\prime}=1.95\right)$, Sinaloa $\left(H^{\prime}=1.99\right)$ and Jalisco $\left(H^{\prime}=2.01\right)$ and highest in Chiapas $\left(H^{\prime}=2.66\right)$. In total, 10 orders, 57 families, 166 genera and 247 species of aquatic insects were identified in samples. Aquatic insect diversity was highest in Chiapas, Oaxaca and Michoacán $\left(H^{\prime}=3.60-3.75\right)$. Mosquito larval/pupal abundance was not correlated with that of predatory Coleoptera and Hemiptera.

Conclusion: This represents the first update on the diversity and geographic distribution of the mosquitoes and aquatic insects of Mexico in over five decades. This information has been cataloged in Mexico's National Biodiversity Information System (SNIB-CONABIO) for public inspection.
\end{abstract}

\section{Background}

Vector-borne diseases transmitted by mosquitoes of the family Culicidae are responsible for $\sim 1.4$ million deaths per year [1] and $17 \%$ of all infectious diseases worldwide [2]. The principal pathogens transmitted by these vectors include viruses (dengue, yellow fever, equine encephalitis, etc.), protozoa (e.g., those causing malaria), and nematodes (e.g. those causing filariasis) [3]. Overall, fewer than 150 species of the genera Anopheles, Aedes and Culex, are the indirect cause of morbidity and mortality among humans, more than any other group of organisms [4].

\footnotetext{
* Correspondence: trevor.inecol@gmail.com

${ }^{3}$ Instituto de Ecología AC, Xalapa, Veracruz, Mexico

Full list of author information is available at the end of the article
}

To date, between 18 and 20 genera and 225-247 species of mosquitoes have been reported from Mexico [5-7]. However, only the subfamilies Anophelinae and Culicinae include vector species of medical or veterinary importance, especially those from the genera: Aedes, Anopheles, Culex, Haemagogus, Mansonia, Sabethes, Psorophora, and Coquillettidia [8,9]. These species oviposit in a wide range of aquatic habitats that also harbor numerous species of aquatic insects and plant species with which they interact. Species interactions are central to the ecology of any habitat, including those of mosquitoes [10]. Identification of the habitats selected as oviposition sites is of clear relevance to mosquito surveillance programs as these habitats are also targeted by mosquito control measures involving habitat elimination or larviciding activities [11]. 
Studies on the bionomics of major mosquito vectors in Mexico have been made [12-16], but few studies have addressed their interactions with other organisms, particularly those involving aquatic insects associated with their oviposition sites [17-21]. Overall 13 orders of insects include species with aquatic or semi-aquatic stages, representing $>95 \%$ of macroinvertebrate species present in aquatic habitats [22,23]. Aquatic insects play key roles in the ecology of aquatic ecosystems and together with other invertebrates, exert an important influence on nutrient cycles and the structure of trophic webs [24]. Apart from their use as biological indicators to evaluate water quality [25], aquatic insects, particularly predatory insects, can play an important role in the biological control of larval and pupal mosquito populations [26].

In this study we describe a comprehensive analysis on the richness, diversity and geographical distribution of mosquitoes in Mexico and the aquatic insects associated with their oviposition sites. The entomological surveys in this study were restricted to the oviposition and immature development sites of mosquitoes because information on the diversity and distribution of endemic vector species is essential to develop vector monitoring and control strategies, which depend on the identity of mosquito species present in each state for effective implementation. This is because public health programs aimed at vector control are decided on a state-by-state basis, depending on state administration budgets and the perceived importance of mosquito control measures and vector borne diseases in each of the 31 states of Mexico. Similarly, this type of baseline information allows detection of changes in the distribution or abundance of species and detection of introduced species of vectors that have extended beyond their natural distribution or biogeographic areas (termed invasive species), and which can cause environmental, economic, and human health impacts.

\section{Methods}

Study area

The study area comprised eight states of the Pacific coast of Mexico, namely Sinaloa, Nayarit, Jalisco, Colima, Michoacan, Guerrero, Oaxaca and Chiapas (Figure 1). The climatic conditions along the Pacific coast region were predominantly characterized by a warm humid climate, with average annual temperature between $22^{\circ}$ and $26^{\circ} \mathrm{C}$ and an annual rainfall of 1,000 to $2,000 \mathrm{~mm}$ [27]. The experimental design consisted of three rounds over the eight study states, one per year for about 55 days, the first was from October 13 to December 6, 2007, the second from September 20 to November 13, 2008 and the third from May 30 to July 23, 2009, giving a total of 165 days of sampling. The selection of sampling sites was based on the reports of type localities for native species of mosquitoes [5,8], and available information on the geographical distribution of mosquito species of medical and veterinary importance in the country $[6,17,28-30]$. In each state, temporary and permanent aquatic sites were sampled that represent potential habitats for the development of larval and pupal populations of mosquitoes, such as pools, lakes, streams, rivers, canals, marshes, etc. In addition, visits were made to cemeteries to collect immature stages of mosquitoes in water tanks. The sampling effort varied from 15 to 61 sites per state (Figure 1). Based on area, the most intensively sampled state was Colima with an average of 2.6 samples per $1000 \mathrm{~km}^{2}$, the least sampled state was Guerrero with an average of 0.3 samples per $1000 \mathrm{~km}^{2}$, whereas the average of all states was 0.6 samples per $1000 \mathrm{~km}^{2}$.

\section{Sampling methods and identification}

Mosquitoes recorded in this study were obtained from field collections, and were compared to information from bibliographic records reported in scientific journals for different states in the study area. Larvae and pupae of mosquitoes were collected by using white enameled dippers, (500 $\mathrm{ml}$ capacity), with a flat outer side. All the larvae and pupae from each aquatic site were collected, counted, placed in 8 oz plastic sample bags, transported to the laboratory and then transferred to $1.5 \mathrm{~mL}$ plastic vials for adult emergence. Mosquitoes that emerged were placed in perforated plastic vials and were stored in plastic containers with silica gel for transport to the laboratories of the Regional Center for Public Health Research, National Public Health Institute (CRISP-INSP), Tapachula, Chiapas. Adult mosquitoes were identified to species using dichotomous keys [31-33].

Aquatic insects were collected using an aquatic entomological net $(24 \times 46 \mathrm{~cm}$ and mesh size $0.9 \mathrm{~mm})$ that was dragged across the bottom and the surface of the water body at each aquatic site. In the case of lakes, rivers and streams an area of $5 \mathrm{~m}^{2}$ was sampled for $5 \mathrm{mi}$ nutes using the traveling kick method [34]. Aquatic insect samples were sorted in white metal trays and were then preserved in $96 \%$ ethanol, taken to the laboratory, and identified to genus and species using the appropriate keys [22,35-42]. Reference specimens of all mosquitoes and aquatic insects were deposited in the entomological collection of CRISP-INSP. Field collection records were entered into the CONABIO Biótica information system 4.5 [43], to create a biodiversity database.

\section{Data analysis}

The abundance of aquatic insects recorded in each state were subjected to multivariate analysis of variance (MANOVA) following $\ln (x+1)$ transformation to normalize the distribution and eliminate zero values. For each of the eight states, $\alpha$ diversity of mosquitoes and aquatic insects was estimated by means of the Shannon index $\left(\mathrm{H}^{\prime}\right)$ 


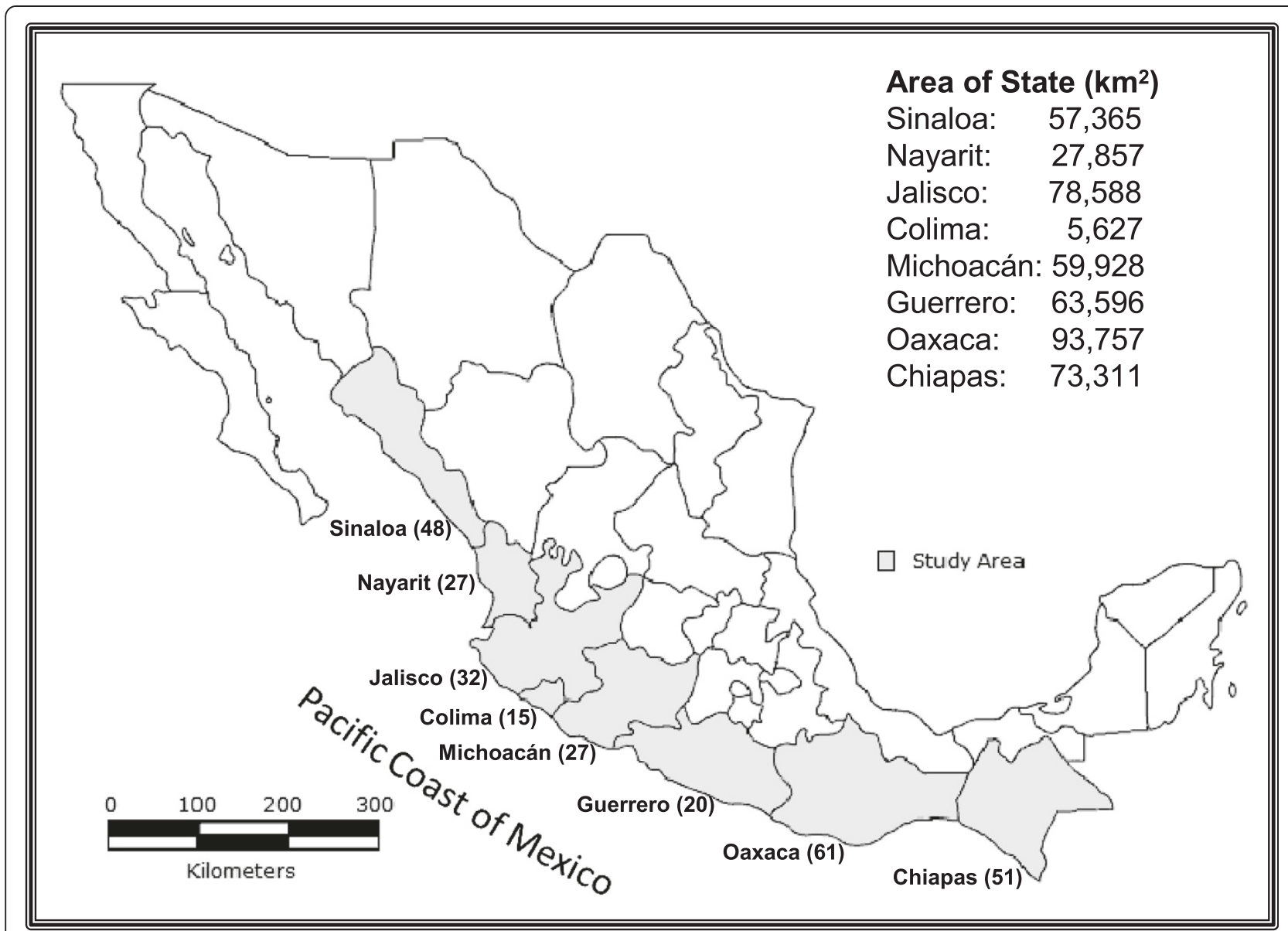

Figure 1 States of the Pacific coast of Mexico included in the study. Values in parentheses indicate total number of samples taken from each state. The surface area $\left(\mathrm{km}^{2}\right)$ of each state is given.

[44]. A randomization test was applied to determine significant differences between $H^{\prime}$ values using the Species Diversity and Richness III (v. 3.0.2) program [45], which is based on the method described by Solow [46]. The accuracy of index values was estimated by jackknifing, which permitted a reduction in the bias in our estimate of the population value and provided a standard error [47]. Confidence intervals for the statistic were calculated by bootstrap with replacement. Differences amongst samples and the communities of mosquitoes and aquatic insects of all the states ( $\beta$ diversity), were estimated by calculating a quantitative similarity index (Morisita-Horn) and cluster analysis $[44,48]$. Correlations of the number of mosquitoes sampled against certain orders of aquatic insects order was performed by Spearman rank correlation in Statistica v.7 (StatSoft Inc. Tulsa, OK).

\section{Results}

\section{Abundance and species richness}

Overall, sampling resulted in the identification of mosquitoes representing 15 genera, 74 species and 4394 individuals distributed among the eight states of the Pacific coast of Mexico. Of the mosquitoes identified to species, $23 \%$ of individuals were from seasonal rain pools followed by streams (22\%), river margins (18\%), lakes and lagoons (14\%), marshes (10\%), irrigation channels (9\%), water tanks (2\%), dams (1\%) and sewers (1\%). At the state level, Chiapas had the highest abundance and richness of culicid taxa, with 1513 individuals (Figure 2), distributed among 14 genera and 54 species, followed by Guerrero and Oaxaca with an abundance of 333 and 495 individuals, respectively, and a richness of 11 and 7 genera, and 38 and 34 species, respectively. The states of Michoacán, Jalisco and Sinaloa had intermediate abundance and richness of mosquitoes that fluctuated between 387-596 individuals from 7 to 9 genera and 21 to 27 species. The states with the lowest abundance and richness of taxa were Colima and Nayarit, with abundance and richness values less than half those observed in Chiapas (Figure 2).

Overall, the most abundant mosquito species were Anopheles pseudopunctipennis, Aedes aegypti and An. 


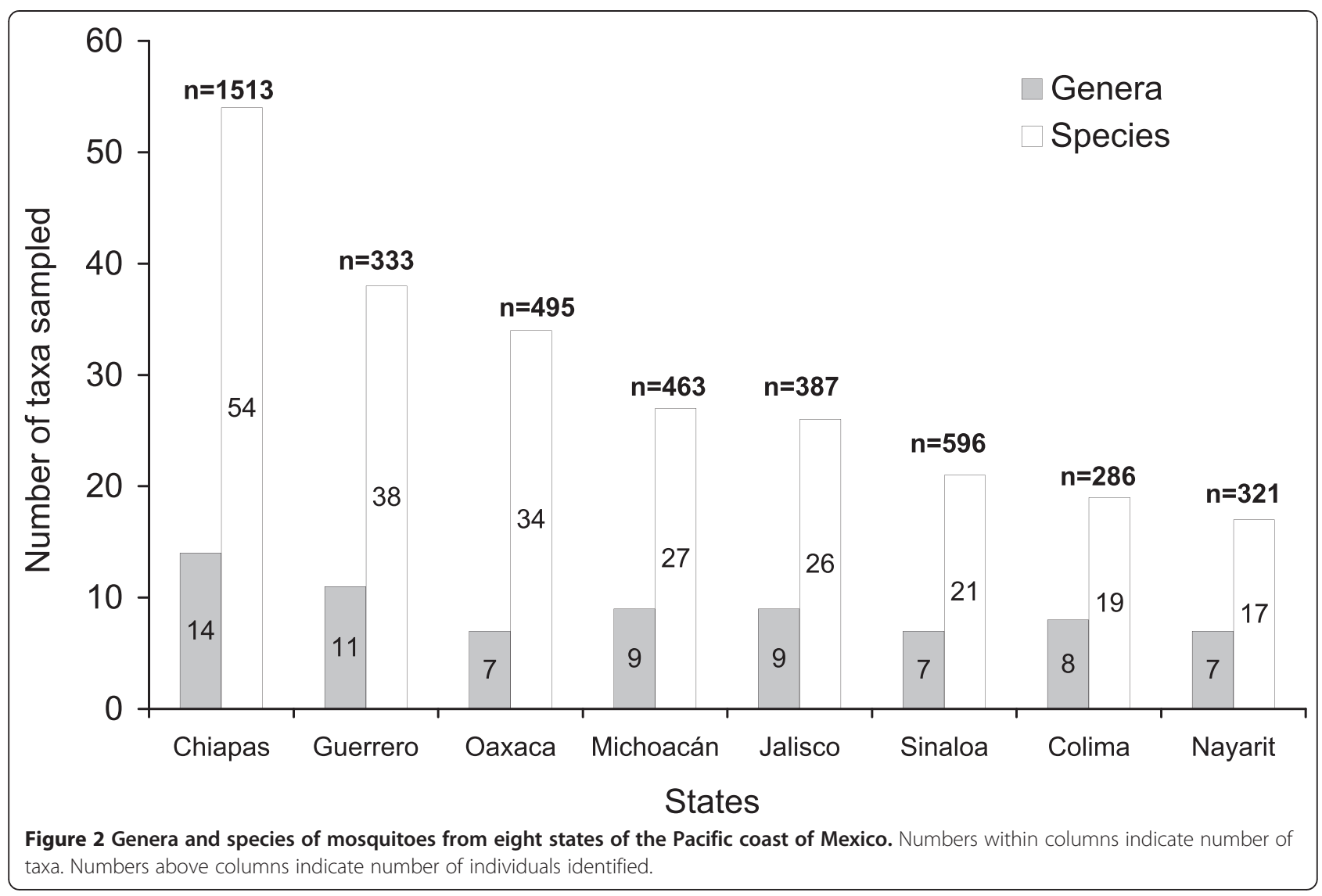

albimanus, with 747 (17.0\%), 664 (15.1\%) and 646 (14.7\%) individuals sampled, respectively (Additional file 1: Table S1). These species represented $47 \%$ of the total mosquitoes collected and were recorded in all eight states of the study area. Additional species that were common and widely distributed in the eight states were Ochlerotatus taeniorhynchus, Culex quinquefasciatus, Cx. coronator, and $C x$. nigripalpus, which together represented $>20 \%$ of the specimens collected. In contrast, the distribution of a number of rare species was restricted to a single state. For example, Ae. albopictus, Ae. angustivitatus, An. darlingi, An. gabaldoni, An. neivai, An. neomaculipalpus, An. vestitipennis, Coquillettidia venesuelensis, and Co. nigricans were collected only in Chiapas. Similarly, Och. infirmatus was only identified in Guerrero, Och. hastatus was collected only in Colima, Psorophora mathesoni was restricted to Jalisco, and Uranotenia orthodoxa was identified only in samples from Michoacán (Additional file 1: Table S1). Overall, Anopheles mosquitoes were the most abundant with 1749 individuals and 19 species, representing almost $40 \%$ of the identified mosquitoes, followed by the genus Culex with 909 individuals (21\%) and 12 species, and Ochlerotatus with 618 individuals (14\%) and 9 species. The remaining 25\% (1118 individuals) included 18 genera and 34 additional species of mosquitoes (Additional file 1: Table S1).
Due to their importance in the transmission of malaria, anopheline mosquitoes were considered to be of particular interest. In total, 18 species of anophelines were recorded during the development of this study (Table 1), and the state of Chiapas had the highest abundance and richness, with 941 individuals, and 15 species, followed by Michoacán and Oaxaca with 48 and 216 individuals, and 9 and 8 species, respectively. Only 5 species were registered in the states of Guerrero, Jalisco and Nayarit with 170, 70 and 95 individuals, respectively. The states with the least species richness were Colima and Sinaloa with 3 species, and 55 and 154 individuals, respectively (Table 1; Additional file 1: Table S1).

\section{Abundance and taxa richness of aquatic insects}

A total of 5233 individuals of aquatic insects were collected, identified and assigned to a total of 10 orders, 57 families, 166 genera and 247 species (Table 2). Significant differences were detected in the abundance of species between collections made in different states along the Pacific coast (MANOVA, Pillai's Trace: $F_{8,240}=7.683$; $P<0.0001)$. The order Coleoptera had the highest abundance of aquatic insects with 1922 individuals and 112 species, followed by the orders Odonata and Hemiptera, with 1128 and 805 individuals, and 64 and 40 species, respectively. Trichoptera, Ephemeroptera, Plecoptera and 
Table 1 Mosquito species recorded from eight states of the Pacific coast of Mexico in previous studies and the present study

\begin{tabular}{|c|c|c|c|c|c|c|c|c|}
\hline \multirow{2}{*}{\multicolumn{9}{|c|}{ Sinaloa Nayarit Jalisco Colima Michoacán Guerrero Oaxaca Chiapas }} \\
\hline & & & & & & & & \\
\hline Aedes aegypti & $\mathbf{x}^{*}$ & $\mathrm{x}^{*}$ & $\mathbf{x}^{*}$ & $\mathrm{x}^{*}$ & $\mathbf{x}^{*}$ & $\mathbf{x}^{*}$ & $\mathbf{x}^{*}$ & $\mathbf{x}^{*}$ \\
\hline Aedes albopictus & & & & & & & & * \\
\hline Aedes angustivittatus & & & & & & & & * \\
\hline Aedes atropalpus & & & & $\mathrm{x}$ & $\mathbf{x}$ & $\mathrm{x}$ & $\mathbf{x}$ & $\mathrm{x}$ \\
\hline Aedes knabi & & & & & & & $\mathbf{x}$ & \\
\hline Aedes quadrivittatus & & & & & & & $\mathbf{x}$ & $\mathrm{x}$ \\
\hline Aedes sexlineatus & & & & & & & & $x$ \\
\hline Aedes vexans & & & $\mathrm{x}^{*}$ & & $\mathrm{x}^{*}$ & & & \\
\hline Aedes terrens & & & $x^{*}$ & & $x^{*}$ & $\neq^{*}$ & $x^{*}$ & $x^{*}$ \\
\hline Aedeomyia squamipennis & & & & & & $x \neq^{*}$ & & $*$ \\
\hline Coquillettidia nigricans & & & & & & & & * \\
\hline Coquillettidia venezuelensis & & & & & & & & $*$ \\
\hline Culex apicalis & & & & & & & $x$ & \\
\hline Culex arizonensis & & & & & & & & $\mathbf{x}$ \\
\hline Culex bidens & $\mathrm{x}$ & & & & & & & $\mathbf{x}$ \\
\hline Culex bigoti & & & & & $x$ & $\mathbf{x}$ & $x$ & $\mathbf{x}$ \\
\hline Culex bihaicolus & & & & & & & & $\mathbf{x}$ \\
\hline Culex chidesteri & & & & & & $x \neq^{*}$ & * & \\
\hline Culex conspirator & $x^{*}$ & & & & & $\neq^{*}$ & $\mathbf{x}^{*}$ & $\mathbf{x}^{*}$ \\
\hline Culex corniger & $x^{*}$ & & & & & $\neq^{*}$ & $x^{*}$ & $\mathbf{x}^{*}$ \\
\hline Culex coronator & $x^{*}$ & * & $\mathrm{x}^{*}$ & $\mathrm{x}^{*}$ & $\mathbf{x}^{*}$ & $x \neq *$ & $\mathrm{x}^{*}$ & $\mathrm{x}^{*}$ \\
\hline Culex declarator & & & & & & $\neq$ & & \\
\hline Culex derivator & & & & & & & & $\mathbf{x}$ \\
\hline Culex educator & & & & & & $\neq$ & & \\
\hline Culex elevator & & & & & & & & $\mathrm{x}$ \\
\hline Culex erraticus & $x^{*}$ & * & * & * & * & $x \neq *$ & $x^{*}$ & * \\
\hline Culex herythrothorax & & & & & $\mathbf{x}$ & & & \\
\hline Culex inflictus & & & & $\mathrm{x}$ & & & & \\
\hline Culex inpatiens & & & & & & & & $\mathbf{x}$ \\
\hline Culex interrogator & $x^{*}$ & & & & $x^{*}$ & * & $x^{*}$ & $\mathbf{x}^{*}$ \\
\hline Culex iolambdis & & & $\mathbf{x}^{*}$ & & & $\ddagger^{*}$ & $\mathbf{x}^{*}$ & $\mathbf{x}^{*}$ \\
\hline Culex maccrackenae & & & & & & & $x$ & $x$ \\
\hline Culex mutator & & & & & & & & $x$ \\
\hline Culex nigripalpus & $x^{*}$ & $x^{*}$ & $\mathbf{x}^{*}$ & & & $x \neq^{*}$ & $x^{*}$ & $\mathbf{x}^{*}$ \\
\hline Culex peccator & & & & & & $\neq$ & & \\
\hline Culex pilosus & & & & & & & & $x$ \\
\hline Culex pinarocampa & & & & & & $\mathrm{x}$ & $\mathrm{x}$ & \\
\hline Culex quinquefasciatus & $x^{*}$ & * & $*$ & $\mathbf{x}^{*}$ & * & $\mathbf{x} \ddagger^{*}$ & $\mathbf{x}^{*}$ & $\mathbf{x}^{*}$ \\
\hline Culex restrictor & & & & & & $*$ & $x^{*}$ & $\mathbf{x}^{*}$ \\
\hline Culex restuans & & & & & $x$ & & & \\
\hline Culex salinarius & & & & & & & $\mathrm{x}$ & \\
\hline Culex stigmatosoma & $\mathrm{x}^{*}$ & * & $\mathbf{x}^{*}$ & * & $x^{*}$ & $x^{*}$ & $\mathrm{x}^{*}$ & $\mathrm{x}^{*}$ \\
\hline
\end{tabular}


Table 1 Mosquito species recorded from eight states of the Pacific coast of Mexico in previous studies and the present study (Continued)

\begin{tabular}{|c|c|c|c|c|c|c|c|c|}
\hline Culex taeniopus & & & & & & $\neq$ & & \\
\hline Culex tarsalis & $\mathbf{x}$ & & & & & $\mathbf{x}$ & & $\mathbf{x}$ \\
\hline Culex thriambus & $x^{*}$ & & & & $\mathbf{x}$ & $\ddagger^{*}$ & $x$ & $\mathbf{x}$ \\
\hline Culex trifidus & & & & & & $x$ & & \\
\hline Culex virgultus & & & & & $\mathbf{x}$ & $\neq$ & $x$ & $\mathbf{x}$ \\
\hline Culiseta particeps & & & & & $*$ & & & \\
\hline Deinocerites belkini & & & * & & & $\#^{*}$ & & \\
\hline Deinocerites epitedeus & $\mathbf{x}$ & $\mathbf{x}$ & & & & & & \\
\hline Deinocerites howardii & & & $*$ & & & & & * \\
\hline Deinocerites pseudes & & & $\mathbf{x}^{*}$ & $*$ & & $x \neq^{*}$ & & \\
\hline Haemagogus equinus & & & $\mathbf{x}^{*}$ & $*$ & $\mathbf{x}^{*}$ & $x \neq^{*}$ & $\mathbf{x}^{*}$ & $x^{*}$ \\
\hline Haemagogus mesodentatus & & & & & & & & $\mathbf{x}$ \\
\hline Limatus durhamii & & & & & & & & $x^{*}$ \\
\hline Mansonia dyari & & * & * & & & & & \\
\hline Mansonia indubitans & & & & & & & $\mathbf{x}^{*}$ & $x^{*}$ \\
\hline Mansonia nigricans & & & & & & $\neq^{*}$ & & \\
\hline Mansonia titillans & $*$ & $\mathbf{x}$ & & $x$ & & $\mathbf{x} \neq^{*}$ & & $x^{*}$ \\
\hline Mansonia venezuelensis & & & & & & & & $\mathbf{x}$ \\
\hline Ochlerotatus angustivittatus & & & & & & $\ddagger^{*}$ & $\mathbf{x}^{*}$ & $x^{*}$ \\
\hline Ochlerotatus atropalpus & & & & $*$ & $*$ & $\ddagger^{*}$ & $*$ & * \\
\hline Ochlerotatus euplocamus & & & & & & & $x$ & \\
\hline Ochlerotatus epactius & $*$ & * & * & $*$ & $*$ & & & * \\
\hline Ochlerotatus infirmatus & & & & & & $\ddagger^{*}$ & & \\
\hline Ochlerotatus hastatus & & & & $\mathbf{x}^{*}$ & & & & \\
\hline Ochlerotatus nigromacul & & & & & & $x$ & & \\
\hline Ochlerotatus podographicus & & $*$ & & & & & & * \\
\hline Ochlerotatus scapularis & & & * & & & $\mathbf{x}^{*}$ & & $x^{*}$ \\
\hline Ochlerotatus serratus & & & $\mathbf{x}$ & & & & & $\mathbf{x}$ \\
\hline Ochlerotatus shannoni & & & & & $x$ & & & \\
\hline Ochlerotatus stigmaticus & & & & & & & $x$ & \\
\hline Ochlerotatus taeniorhynchus & $\mathrm{x}^{*}$ & $x^{*}$ & $x^{*}$ & $\mathbf{x}^{*}$ & $x^{*}$ & $x \neq^{*}$ & $\mathbf{x}^{*}$ & $x^{*}$ \\
\hline Ochlerotatus thelcter & $\mathbf{x}$ & & & & & & & \\
\hline Ochlerotatus trivittatus & & & & & $x^{*}$ & $\mathbf{x} \neq$ & $\mathbf{x}^{*}$ & \\
\hline Ochlerotatus torilis & & & & & & $\mathbf{x}$ & & \\
\hline Psorophora champerico & $\mathbf{x}$ & & & & & & & $\mathbf{x}$ \\
\hline Psorophora ciliata & & & & & & & $x$ & $\mathbf{x}$ \\
\hline Psorophora cilipes & & & & & & $\neq$ & & \\
\hline Psorophora confinnis & $\mathbf{x}^{*}$ & & & & $\mathbf{x}$ & & $\mathbf{x}^{*}$ & $x^{*}$ \\
\hline Psorophora cyanescens & $\mathbf{x}^{*}$ & & $\mathbf{x}^{*}$ & & & $\ddagger^{*}$ & $\mathbf{x}^{*}$ & $x^{*}$ \\
\hline Psorophora discolor & & & & & & & $\mathbf{x}^{*}$ & $x^{*}$ \\
\hline Psorophora ferox & & & & & $\mathbf{x}^{*}$ & $\ddagger^{*}$ & $\mathbf{x}^{*}$ & $x^{*}$ \\
\hline Psorophora howardii & & & & & & $\mathbf{x}$ & & $\mathbf{x}$ \\
\hline Psorophora lutzii & & & & & & $\ddagger^{*}$ & $x^{*}$ & $x^{*}$ \\
\hline Psorophora mexicana & & & & & & & $x$ & \\
\hline
\end{tabular}


Table 1 Mosquito species recorded from eight states of the Pacific coast of Mexico in previous studies and the present study (Continued)

\begin{tabular}{|c|c|c|c|c|c|c|c|c|}
\hline Psorophora mathesoni & & & * & & & & & \\
\hline Psorophora varipes & & & $\mathbf{x}^{*}$ & $x^{*}$ & & $\ddagger^{*}$ & $\mathbf{x}^{*}$ & * \\
\hline Psorophora virescens & & $x^{*}$ & & $x^{*}$ & & $x^{*}$ & $x^{*}$ & \\
\hline Sabethes chloropterus & & & & & $\mathbf{x}^{*}$ & & & $x^{*}$ \\
\hline Uranotaenia coatzacoalcos & & & & & $\mathbf{x}$ & & $\mathbf{x}$ & $\mathbf{x}$ \\
\hline Uranotaenia geometrica & & & & & & & & $\mathbf{x}$ \\
\hline Uranotaenia lowii & $\mathbf{x}^{*}$ & * & * & * & & $\ddagger^{*}$ & & * \\
\hline Uranotaenia orthodoxa & & & & & * & & & \\
\hline Uranotaenia pulcherrima & & & & & & $x$ & & \\
\hline Uranotaenia socialis & * & & & * & & & & \\
\hline Uranotaenia sapphirina & $\mathbf{x}^{*}$ & & & $\mathbf{x}^{*}$ & $x^{*}$ & $x \neq^{*}$ & & $x^{*}$ \\
\hline Wyeomyia arthrostigma & & & & & & & & $\mathbf{x}$ \\
\hline Wyeomyia celaenocephala & & & & & & & & $\mathbf{x}$ \\
\hline Wyeomyia jocosa & & & & & & & & $\mathbf{x}$ \\
\hline Wyeomyia mitchelli & & & & & & & & $\mathrm{x}^{*}$ \\
\hline Wyeomyia personata & & & & & & $\ddagger^{*}$ & & $\mathbf{x}^{*}$ \\
\hline \multicolumn{9}{|l|}{ (B) Anophelines } \\
\hline Anopheles albimanus & $x^{*}$ & $x^{*}$ & $\mathbf{x}^{*}$ & $x^{*}$ & $x^{*}$ & $x \neq^{*}$ & $\mathbf{x}^{*}$ & $\mathrm{x}^{*}$ \\
\hline Anopheles apicimacula & & & & & $\mathbf{x}^{*}$ & & & $\mathbf{x}^{*}$ \\
\hline Anopheles argyritarsis & & $\mathbf{x}^{*}$ & & & $\mathbf{x}^{*}$ & & $\mathbf{x}^{*}$ & $\mathrm{x}^{*}$ \\
\hline Anopheles aztecus & & & $\mathbf{x}^{*}$ & & $x^{*}$ & & & \\
\hline Anopheles crucians & & & & * & & $\ddagger^{*}$ & $*$ & $*$ \\
\hline Anopheles darlingi & & & & & & & & $\mathbf{x}^{*}$ \\
\hline Anopheles eiseni & & & & & $x^{*}$ & $x^{*}$ & $\mathbf{x}^{*}$ & $\mathbf{x}^{*}$ \\
\hline Anopheles franciscanus & & & * & & & & * & $*$ \\
\hline Anopheles freeborni & & & & & * & & & \\
\hline Anopheles gabaldoni & & & & & & & & $\mathbf{x}^{*}$ \\
\hline Anopheles hectoris & & & & & & & & $x^{*}$ \\
\hline Anopheles neivai & & & & & & & & $\mathbf{x}^{*}$ \\
\hline Anopheles neomaculipalpus & & & & & & & & $x^{*}$ \\
\hline Anopheles parapunctipennis & & & & & & & $\mathbf{x}^{*}$ & $\mathbf{x}^{*}$ \\
\hline Anopheles pseudopunctipennis & $\mathbf{x}^{*}$ & $x^{*}$ & $\mathbf{x}^{*}$ & $x^{*}$ & $x^{*}$ & $x \neq^{*}$ & $\mathbf{x}^{*}$ & $x^{*}$ \\
\hline Anopheles punctimacula & * & $x^{*}$ & & & $\mathbf{x}^{*}$ & $x \ddagger^{*}$ & $\mathbf{x}^{*}$ & $\mathbf{x}^{*}$ \\
\hline Anopheles punctipennis & & * & * & & $x^{*}$ & & & \\
\hline Anopheles vestitipennis & & & & & & & & $\mathbf{x}^{*}$ \\
\hline Anopheles xelajuensis & & & & & & & $\mathbf{x}$ & \\
\hline Species records & Sinaloa & Nayarit & Jalisco & Colima & Michoacán & Guerrero & Oaxaca & Chiapas \\
\hline No. species from previous records $(\mathbf{x})[28,29]$ & 22 & 10 & 16 & 13 & 29 & $52 \neq$ & 46 & 67 \\
\hline No. species in present study $(*)$ & 21 & 17 & 26 & 19 & 27 & 38 & 34 & 54 \\
\hline Previous species records confirmed in present study & 17 & 8 & 15 & 10 & 20 & 36 & 30 & 40 \\
\hline Species exclusive to this study & 4 & 9 & 11 & 9 & 7 & 2 & 4 & 14 \\
\hline Total number of species per state & 26 & 19 & 27 & 22 & 36 & 54 & 50 & 81 \\
\hline
\end{tabular}

$\mathbf{x}$ Indicates species reported in previous studies $[28,29]$.

*Indicates species reported in the present study.

\#Double dagger symbol indicates species records from Guerrero state [17]. 
Table 2 Aquatic insect species associated with the oviposition sites of mosquitoes and their presence in each of eight states* along the Pacific coast of Mexico

\begin{tabular}{|c|c|c|c|c|c|c|c|c|c|}
\hline Species & Distribution & Species & Distribution & Species & Distribution & Species & Distribution & Species & Distribution \\
\hline Abedus ovatus & Ch, G, O & Crenitis sp. & $C, M$ & $\begin{array}{l}\text { Hesperagrion } \\
\text { heterodoxum }\end{array}$ & O & Macrelmis sp. & $\mathrm{Ch}$ & Phyllogomphoides sp. & $\mathrm{Ch}$ \\
\hline Ablabesmyia sp. & C & Cryphocricos sp. & $\mathrm{Ch}$ & $\begin{array}{l}\text { Hesperocorixia } \\
\text { vulgaris }\end{array}$ & $\mathrm{Ch}$ & Macronychus sp. & $\mathrm{Ch}$ & $\begin{array}{l}\text { Phyllogomphoides } \\
\text { suasus }\end{array}$ & Ch, G, N \\
\hline Acilius sp. & $J, M, N$ & Curicta howardi & 0 & $\begin{array}{l}\text { Hetaerina } \\
\text { cruentata }\end{array}$ & $\mathrm{Ch}, \mathrm{G}, \mathrm{N}, \mathrm{O}$ & Macrothemis inacuta & $\mathrm{G}, \mathrm{N}, \mathrm{O}$ & Platyvelia sp. & $\mathrm{Ch}$ \\
\hline Acneus sp. & $\mathrm{Ch}$ & Curicta sp. & $\mathrm{J}, \mathrm{S}$ & $\begin{array}{l}\text { Hetaerina } \\
\text { vulnerata }\end{array}$ & $J, N, S$ & $\begin{array}{l}\text { Macrothemis } \\
\text { pseudimitans }\end{array}$ & $\mathrm{Ch}, \mathrm{G}, \mathrm{N}, \mathrm{O}, \mathrm{S}$ & Potamyia flava & $\mathrm{Ch}$ \\
\hline $\begin{array}{l}\text { Rhionaeschna } \\
\text { multicolor }\end{array}$ & $J, M$ & Cybister sp. & $\begin{array}{l}C, G, J, M \\
N, O, S\end{array}$ & $\begin{array}{l}\text { Hetarina } \\
\text { americana }\end{array}$ & $G, J, N, O$ & Macrothemis ultima & $\mathrm{J}, \mathrm{S}$ & Progomphus clendoni & $\mathrm{Ch}$ \\
\hline Agabinus sp. & J & Cylloepus sp. & $\mathrm{Ch}$ & $\begin{array}{l}\text { Heteragrion } \\
\text { tricellulare }\end{array}$ & $\mathrm{Ch}, \mathrm{O}$ & Macrovelia hornii & $\mathrm{Ch}$ & Progomphus sp. & $\mathrm{Ch}$ \\
\hline Agabus sp. & $\mathrm{Ch}$ & Cyphon sp. & $J, N, S$ & Heterelmis obesa & $\mathrm{Ch}, \mathrm{O}$ & Macrovelia sp. & M & Psephenus sp. & $\mathrm{Ch}$ \\
\hline Ambrysus mormon & $C, C h, J, S$ & Derallus rudis & $\mathrm{C}, \mathrm{Ch}, \mathrm{O}, \mathrm{S}$ & Hydaticus sp. & $C, C h, J, M, S$ & Megadytes sp. & $J, M$ & Pseudoleon superbus & $J, M, S$ \\
\hline Ambrysus sp. & $\begin{array}{l}\mathrm{C}, \mathrm{G}, \mathrm{M} \\
\mathrm{N}, \mathrm{O}, \mathrm{S}\end{array}$ & Derovatellus sp. & S & Hydraena sp. & J & Mesovelia mulsanti & $C, N$ & Ranatra sp. & $\begin{array}{l}\text { C, Ch, G, J, M, } \\
\text { N, O, S }\end{array}$ \\
\hline Anacaena suturalis & $C, J, M, S$ & Desmopachria sp. & $C, J, M, N$ & $\begin{array}{l}\text { Hydrobiomorpha } \\
\text { casta }\end{array}$ & J. $M, O, S$ & Mesovelia sp. & $C h, C, J, M$ & Rhagovelia sp. & $\mathrm{C}, \mathrm{Ch}, \mathrm{O}$ \\
\hline Anacroneuria sp. & $\mathrm{Ch}$ & $\begin{array}{l}\text { Desmopachria } \\
\text { striola }\end{array}$ & S & $\begin{array}{l}\text { Hydrocanthus } \\
\text { oblongus }\end{array}$ & $\mathrm{J}, \mathrm{N}$ & Metrobates sp. & $\mathrm{Ch}$ & Rhantus calidus & $\mathrm{O}$ \\
\hline Anax amazili & $\mathrm{C}, \mathrm{Ch}, \mathrm{N}, \mathrm{S}$ & Dicranopselaphus sp. & $\mathrm{Ch}$ & Hydrocanthus sp. & $\begin{array}{l}\mathrm{C}, \mathrm{G}, \mathrm{J}, \mathrm{M}, \\
\mathrm{N}, \mathrm{O}, \mathrm{S}\end{array}$ & Micrathyria aequalis & $C h, N, J$ & $\begin{array}{l}\text { Rhantus } \\
\text { gutticollis }\end{array}$ & $\mathrm{O}, \mathrm{S}$ \\
\hline Anax junius & $J, M$ & Diglotta sp. & $\mathrm{Ch}$ & Hydrochara sp. & $\mathrm{Ch}$ & Micrathyria hagenii & $C, M$ & Rhantus sp. & $\mathrm{O}, \mathrm{S}$ \\
\hline Antocha sp. & $\mathrm{Ch}$ & Dineutus ciliatus & $\mathrm{G}, \mathrm{O}$ & Hydrochus sp. & $C, J, M, N, S$ & $\begin{array}{l}\text { Microcylloepus } \\
\text { inaequalis }\end{array}$ & $\mathrm{Ch}, \mathrm{G}$ & Rhionaeschna psilus & M \\
\hline Apteraliplus sp. & $C, S$ & Dineutus discolor & $\mathrm{Ch}$ & Hydroisotoma sp. & $\mathrm{Ch}$ & $\begin{array}{l}\text { Microvelia } \\
\text { beameri }\end{array}$ & $\mathrm{Ch}$ & Rhionaeschna sp. & $M, S$ \\
\hline Aquarius sp. & J & Dixella sp. & $\mathrm{Ch}$ & $\begin{array}{l}\text { Hydrometra } \\
\text { australis }\end{array}$ & $\mathrm{Ch}$ & Microvelia sp. & $C, C h, J, M, N, O$ & Scirtes sp. & J \\
\hline Archilestes sp. & M & Dryops sp. & $\mathrm{Ch}$ & Hydrometra sp. & C, Ch, N & Neocylloepus sp. & Ch, M & Simulium sp. & Ch, M \\
\hline Argia anceps & $\mathrm{N}$ & Dubiraphia sp. & $\mathrm{Ch}$ & $\begin{array}{l}\text { Hydrophilus } \\
\text { insularis }\end{array}$ & 0 & Neoperla sp. & $C, C h, G, J, M, N, S$ & Steinovelia stagnalis & $\mathrm{Ch}$ \\
\hline Argia fissa & $M, N, S$ & Dytiscus sp. & J, M & $\begin{array}{l}\text { Hydrophilus } \\
\text { smaragdinus }\end{array}$ & 0 & $\begin{array}{l}\text { Nerthra } \\
\text { mexicana }\end{array}$ & $\mathrm{Ch}, \mathrm{O}$ & Stenelmis sp. & $\mathrm{Ch}$ \\
\hline Argia oculata & $C, C h, G, N, O$ & Enallagma sp. & $C, J$ & Hydrophilus sp. & $G, S$ & Nerthra sp. & $\mathrm{C}, \mathrm{O}$ & Stenus sp. & $\mathrm{Ch}$ \\
\hline Argia oenea & $C h, G, O$ & Enochrus blatchleyi & 0 & $\begin{array}{l}\text { Hydrophilus } \\
\text { triangularis }\end{array}$ & $\mathrm{Ch}$ & Notomicrus sp. & C & Stratiomys sp. & $J, S$ \\
\hline
\end{tabular}


Table 2 Aquatic insect species associated with the oviposition sites of mosquitoes and their presence in each of eight states* along the Pacific coast of Mexico (Continued)

\begin{tabular}{|c|c|c|c|c|c|c|c|c|c|}
\hline Argia pulla & $\begin{array}{l}\text { C, Ch, G, M, } \\
\text { N, O, S }\end{array}$ & Enochrus mexicanus & $\begin{array}{l}\text { C, Ch, J, M, } \\
\text { N, O, S }\end{array}$ & $\begin{array}{l}\text { Hydropsyche } \\
\text { betteni }\end{array}$ & $\mathrm{Ch}$ & Notonecta sp. & $\mathrm{G}, \mathrm{J}, \mathrm{M}, \mathrm{O}, \mathrm{S}$ & Suphis sp. & $J, M, S$ \\
\hline Atopsyche sp. & Ch & Enochrus ochraceus & $\mathrm{O}$ & Hydropsyche sp. & Ch, M & Ochterus sp. & Ch & $\begin{array}{l}\text { Suphisellus } \\
\text { lineatus }\end{array}$ & $C, N, S$ \\
\hline Baetis sp. & $\mathrm{Ch}$ & $\begin{array}{l}\text { Enochrus } \\
\text { pseudochraceus }\end{array}$ & M & Hydroscapha sp. & $S$ & Optioservus sp. & $\mathrm{Ch}$ & Suphisellus sp. & $\mathrm{J}, \mathrm{M}, \mathrm{N}, \mathrm{O}$ \\
\hline Baetodes sp. & $\mathrm{Ch}$ & Enochrus pygmaeus & $C, G, J, M, O, S$ & Ischnura capreolus & $\mathrm{C}, \mathrm{Ch}, \mathrm{N}$ & Ordobrevia sp. & Ch, M & $\begin{array}{l}\text { Sympetrum } \\
\text { illotum }\end{array}$ & $M, S$ \\
\hline Barbaetis sp. & $\mathrm{Ch}$ & $\begin{array}{l}\text { Erpetogomphus } \\
\text { elaps }\end{array}$ & Ch, G, N, O & Ischnura demorsa & $\begin{array}{l}C_{1} J, M \\
N, O, S\end{array}$ & $\begin{array}{l}\text { Orthemis } \\
\text { ferruginea }\end{array}$ & $J, M, S$ & Tabanus sp. & M \\
\hline Belostoma sp. & $C, C h, J, S$ & $\begin{array}{l}\text { Erpetogomphus } \\
\text { eutainia }\end{array}$ & $C h, G, O$ & Ischnura hastata & $\begin{array}{l}\mathrm{Ch}, \mathrm{G}, \mathrm{M} \\
\mathrm{N}, \mathrm{O}, \mathrm{S}\end{array}$ & Orthemis sp. & M & Telebasis salva & $C, J, M, N, S$ \\
\hline Berosus arneti & s & Erpetogomphus sp. & $\mathrm{Ch}$ & Ischnura ramburii & $\begin{array}{l}\text { C, G, J, M, } \\
\text { N, O, S }\end{array}$ & $\begin{array}{l}\text { Pachydiplax } \\
\text { longipennis }\end{array}$ & C & Telebasis sp. & $\mathrm{C}, \mathrm{N}, \mathrm{S}$ \\
\hline Berosus exiguus & $\mathrm{O}$ & Erythemis attala & $C, M, N, O$ & Ischnura sp. & $C, C h, J, M, N, S$ & & & Tenagobia sp. & M \\
\hline $\begin{array}{l}\text { Berosus } \\
\text { infuscatus }\end{array}$ & $\mathrm{O}, \mathrm{S}$ & Erythemis plebeja & $\begin{array}{l}\mathrm{C}, \mathrm{Ch}, \mathrm{G}, \mathrm{J} \\
\mathrm{N}, \mathrm{O}, \mathrm{S}\end{array}$ & Isonychia sp. & $\mathrm{Ch}$ & Pachydrus princeps & $G, M$ & $\begin{array}{l}\text { Thermonectus } \\
\text { basillaris }\end{array}$ & $\mathrm{J}, \mathrm{O}, \mathrm{S}$ \\
\hline Berosus mexicanus & $\begin{array}{l}\mathrm{C}, \mathrm{G}, \mathrm{J}, \mathrm{M}, \\
\mathrm{N}, \mathrm{O}, \mathrm{S}\end{array}$ & Erythemis sp. & $M, N, S$ & Laccobius sp. & $\mathrm{M}, \mathrm{N}$ & Pachydrus sp. & M & $\begin{array}{l}\text { Thermonectus } \\
\text { marmoratus }\end{array}$ & $\mathrm{G}, \mathrm{O}, \mathrm{S}$ \\
\hline Berosus sayi & M & Erythemis vesiculosa & $\mathrm{C}, \mathrm{G}, \mathrm{N}, \mathrm{O}$ & Laccodytes sp. & Ch, C, M, S & $\begin{array}{l}\text { Palaemnema } \\
\text { desiderata }\end{array}$ & $\mathrm{Ch}, \mathrm{O}$ & $\begin{array}{l}\text { Thermonectus } \\
\text { ornaticollis }\end{array}$ & S \\
\hline Bibiocephala grandis & Ch & Erythrodiplax sp. & $C, M$ & Laccophilus fasciatus & $\begin{array}{l}C h, C, G, J, M, \\
N, O, S\end{array}$ & $\begin{array}{l}\text { Pantala } \\
\text { flavescens }\end{array}$ & $\mathrm{C}, \mathrm{Ch}, \mathrm{G}, \mathrm{N}, \mathrm{S}$ & Thermonectus sp. & $\mathrm{Ch}, \mathrm{C}, \mathrm{J}, \mathrm{O}, \mathrm{S}$ \\
\hline Bidessonotus sp. & C & $\begin{array}{l}\text { Erythrodiplax } \\
\text { umbrata }\end{array}$ & $C, G, J, N, O$ & Laccophilus hyalinus & M & $\begin{array}{l}\text { Pantala } \\
\text { hymenaea }\end{array}$ & $J, S$ & Tramea abdominalis & $C, J$ \\
\hline Brachydeutera sp. & s & Gelastocoris oculatus & $J, N$ & Laccophilus maculosus & $G, J, O, S$ & Pantala sp. & Ch. S & Trepobates pictus & $\mathrm{N}$ \\
\hline $\begin{array}{l}\text { Brechmorhoga } \\
\text { mendax }\end{array}$ & $\mathrm{N}$ & Gelastocoris sp. & $M, N, S$ & Laccophilus pictus & $\mathrm{Ch}, \mathrm{G}, \mathrm{J}, \mathrm{O}, \mathrm{S}$ & Paracloeodes sp. & $\mathrm{Ch}$ & Trepobates sp. & $\mathrm{Ch}, \mathrm{C}, \mathrm{J}, \mathrm{N}$ \\
\hline $\begin{array}{l}\text { Brechmorhoga } \\
\text { praecox }\end{array}$ & $\mathrm{Ch}, \mathrm{G}, \mathrm{N}, \mathrm{O}$ & Gerris sp. & $\mathrm{Ch}$ & Laccophilus sp. & $C, C h, J, M, S$ & $\begin{array}{l}\text { Paracymus } \\
\text { armatus }\end{array}$ & $\mathrm{Ch}$ & Triacanthagyna sp. & M \\
\hline Brechmorhoga vivax & Ch & Gonielmis sp. & $\mathrm{Ch}$ & Laccophilus undatus & C & $\begin{array}{l}\text { Paracymus } \\
\text { confusus }\end{array}$ & $\mathrm{O}$ & Trichocorixa sp. & $\mathrm{O}, \mathrm{S}$ \\
\hline Bryothinusa sp. & $\mathrm{Ch}$ & Graphoderus sp. & J & Lara sp. & S & $\begin{array}{l}\text { Paracymus } \\
\text { regularis }\end{array}$ & $\mathrm{C}, \mathrm{Ch}, \mathrm{M}, \mathrm{N}, \mathrm{O}, \mathrm{S}$ & Tricorythodes sp. & $\mathrm{Ch}$ \\
\hline Buenoa sp. & $\begin{array}{l}\text { C, Ch, G, J, M, } \\
\text { N, O, S }\end{array}$ & Graptocorixa sp. & $J, S$ & $\begin{array}{l}\text { Leptobasis } \\
\text { vacillans }\end{array}$ & M & Paradelphomyia sp. & $\mathrm{Ch}$ & Trochopus sp. & Ch \\
\hline $\begin{array}{l}\text { Caloparyphus } \\
\text { greylockensis }\end{array}$ & $\mathrm{Ch}$ & Gyrinus parcus & $\mathrm{O}$ & Leptohyphes sp. & $\mathrm{Ch}$ & Paraleptophlebia sp. & $\mathrm{Ch}$ & Tropisternus blatchleyi & S \\
\hline
\end{tabular}


Table 2 Aquatic insect species associated with the oviposition sites of mosquitoes and their presence in each of eight states* along the Pacific coast of Mexico (Continued)

\begin{tabular}{|c|c|c|c|c|c|c|c|c|c|}
\hline Camelobaetidius sp. & $\mathrm{Ch}$ & Haliplus sp. & $J, M$ & Leptonema sp. & $\mathrm{Ch}$ & Pelocoris sp. & $\begin{array}{l}\text { C, Ch, G, J, M, } \\
\text { N, O, S }\end{array}$ & $\begin{array}{l}\text { Tropisternus } \\
\text { collaris }\end{array}$ & $\begin{array}{l}\mathrm{C}, \mathrm{G}, \mathrm{J}, \mathrm{M}, \\
\mathrm{N}, \mathrm{O}, \mathrm{S}\end{array}$ \\
\hline Celina sp. & J & Hebrus sobrinus & $\mathrm{Ch}$ & Lestes alacer & $\mathrm{M}, \mathrm{O}$ & $\begin{array}{l}\text { Pelonomus } \\
\text { obscurus }\end{array}$ & $\mathrm{O}$ & Tropisternus lateralis & $J, M, O, S$ \\
\hline Ceratopsyche sp. & $\mathrm{Ch}$ & Helichus sp. & $\mathrm{Ch}, \mathrm{S}$ & Lestes tenuatus & $\mathrm{G}, \mathrm{J}, \mathrm{M}, \mathrm{N}, \mathrm{O}$ & Pelonomus sp. & C & $\begin{array}{l}\text { Tropisternus } \\
\text { mixtus }\end{array}$ & $M, N$ \\
\hline Chaetarthria sp. & $M, S$ & Helobata sp. & $\mathrm{Ch}, \mathrm{S}$ & Lestes tikalus & $\mathrm{Ch}, \mathrm{C}, \mathrm{O}$ & $\begin{array}{l}\text { Peltodytes } \\
\text { dietrichi }\end{array}$ & $S$ & Tropisternus paredesi & s \\
\hline Chimarra sp. & Ch & $\begin{array}{l}\text { Helochares } \\
\text { normatus }\end{array}$ & $C, G, S$ & Leucotrichia sp. & $\mathrm{Ch}$ & $\begin{array}{l}\text { Peltodytes } \\
\text { muticus }\end{array}$ & M & Tropisternus sp. & $\begin{array}{l}C, G, J, M, \\
N, O, S\end{array}$ \\
\hline $\begin{array}{l}\text { Copelatus } \\
\text { caelatipennis }\end{array}$ & O & Helochares sallaei & S & Libellula foliata & $\mathrm{Ch}$ & Peltodytes sp. & $S$ & Uvarus sp. & $C, M, S$ \\
\hline Copelatus sp. & $J, M$ & Helophorus sp. & J & Liodessus fuscatus & $\mathrm{G}, \mathrm{O}, \mathrm{S}$ & Perissolestes sp. & M & Zaitzevia sp. & $\mathrm{Ch}$ \\
\hline Corisella decolor & M & Hemerodromia sp. & M & Liodessus sp. & $C, J, M, N, S$ & Perithemis sp. & S & & \\
\hline Corydalus cornutus & C, Ch, M & Henochrus sp. & $\mathrm{Ch}$ & Lipogomphus sp. & $C h, C, J$ & $\begin{array}{l}\text { Petrophila } \\
\text { confusalis }\end{array}$ & $\mathrm{Ch}$ & & \\
\hline
\end{tabular}

${ }^{*} \mathrm{C}=$ Colima, $\mathrm{Ch}=$ Chiapas, $\mathrm{G}=$ Guerrero, $\mathrm{J}=$ Jalisco, $\mathrm{M}=$ Michoacán, $\mathrm{N}=$ Nayarit, $\mathrm{O}=$ Oaxaca, $\mathrm{S}=$ Sinaloa. 
Diptera (not including mosquitoes) presented an intermediate abundance with 523, 331, 283 and 141 individuals, and 8, 9, 2 and 10 species, respectively. The least represented groups in the collections were Collembola with only one individual and one species, and Lepidoptera and Megaloptera, with 30 and 69 individuals respectively, each represented by a single species (Figure 3A and 3B). Chiapas state had the highest taxa richness, with 116 species distributed in 100 genera, 46 families and 10 orders (Figure 4), followed by the states of Sinaloa, Michoacan and Oaxaca, with between 86 and 71 species. The states with the lowest species richness were Jalisco, Colima, Nayarit and Guerrero with between 68 and 41 species collected during the sampling program (Figure 4).

\section{Anopheline diversity}

Shannon index diversity values differed between states $(\delta=0.39-1.06 ; P<0.001)$, and allowed states to be classified into four groups (Figure 5A). The highest diversity values were observed in collections made in the states of Chiapas and Michoacán ( $\mathrm{H}^{\prime}=1.61$ and 1.56, respectively). Collections made in the states of Oaxaca and Jalisco generated index values of 1.21 and 1.10, respectively. A third group of states comprised the states of Nayarit, Colima and Guerrero with diversity values of $0.80,0.72$ and 0.69 , respectively. Finally, the state of Sinaloa had the lowest H' value at 0.54 , which did not differ significantly from the value calculated for the state of Colima. Jackknifing indicated that diversity index values were underestimated by 4.14\% for Anopheles spp. (Table 3). Diversity index findings were consistent with the cluster analysis, which also discriminated four groups depending on their species abundance, with the only difference being that in the analysis of diversity, Sinaloa clustered in a group that comprised Guerrero, Nayarit and Colima (Figure 6A).

\section{Culicid diversity}

Significant differences were detected between culicid diversity values from the eight states. Culicid diversity values fell into one of three groups: first, Chiapas state with a diversity index value of 2.66 that was significantly higher than the values of the seven remaining states. The second group comprised the states of Oaxaca $\left(\mathrm{H}^{\prime}=\right.$ 2.37), Guerrero $\left(H^{\prime}=2.30\right)$ and Nayarit $\left(H^{\prime}=2.22\right)$ with similar diversity values. The third group comprised Jalisco $\left(H^{\prime}=2.01\right)$, Sinaloa $\left(H^{\prime}=1.99\right)$, Colima $\left(H^{\prime}=1.95\right)$ and Michoacán $\left(\mathrm{H}^{\prime}=1.93\right)$ with the lowest diversity values (Figure 5B). Cluster analysis broadly supported these findings, the main difference being that Chiapas clustered with Oaxaca and Guerrero, rather than forming a separate group (Figure 6B). Jackknifing indicated that culicid diversity index values were underestimated by $4.57 \%$ (Table 3 ).

\section{Aquatic insect diversity}

Diversity analyses on aquatic insect samples (Figure 5C), indicated that Chiapas had the highest Shannon index value (3.75), followed by the states of Oaxaca (3.63), Michoacan (3.60). Intermediate $H^{\prime}$ values were estimated for the states of Sinaloa, Guerrero, Jalisco and Nayarit that varied between 3.34 and 3.54. The lowest index value was calculated for the state of Colima (3.22), which was significantly lower than all other values, except that calculated for the state of Nayarit. Jackknifing indicated that $\mathrm{H}^{\prime}$ values were underestimated by $3.34 \%$ for aquatic insects (Table 3). Cluster analysis supported the existence of three diversity groups consisting of Chiapas alone (Figure 6C), the second group consisting of Oaxaca, Michoacan and Sinaloa and the third group consisting of Guerrero, Jalisco, Nayarit and Colima. Minor differences in the placement of states within a particular group were observed, but the overall patterns were in agreement with the calculated diversity index values.

\section{Similarity analyses and correlations}

Morisita-Horn similarity index values for comparison of species composition of mosquitoes among the states of Chiapas-Oaxaca or Chiapas-Guerrero were estimated at $70 \%$ or at $91 \%$ in the comparison of the states of OaxacaGuerrero (Table 4). Similar results were observed in the cluster analysis, in which culicids from these three states clustered in a single group (Figure 6B). Similarity in species composition of mosquitoes among the states of Jalisco, Colima and Sinaloa were close to $70 \%$, whereas comparison of the mosquito composition of Michoacan and Jalisco was extremely high at 92\% (Table 4). These findings were consistent with those of the cluster and diversity analyses that included the states of Jalisco, Colima, Sinaloa and Michoacán within a group according to their species composition or diversity (Figures 5A and 6A).

The presence of mosquito larvae was not significantly correlated with the prevalence of aquatic insect predators of the orders Coleoptera (Spearman's $r_{s}=-0.056, \mathrm{~N}=$ 180 ), or Hemiptera (Spearman's $r_{s}=-0.141, N=80$ ). Additional correlations were not performed as the low numbers of independent points reduced the validity of the correlation, e.g., as was the case for Odonata.

\section{Discussion}

The diversity of mosquito species was determined for each of eight states along 2,500 km of the Pacific coast of Mexico. This represents the most complete mosquito survey of this region to date and markedly expands the information provided by the only previous detailed studies on the mosquito fauna of Mexico performed in the 1950's [28,29]. A large and taxonomically diverse group of aquatic insects associated with mosquito oviposition sites was also identified. 


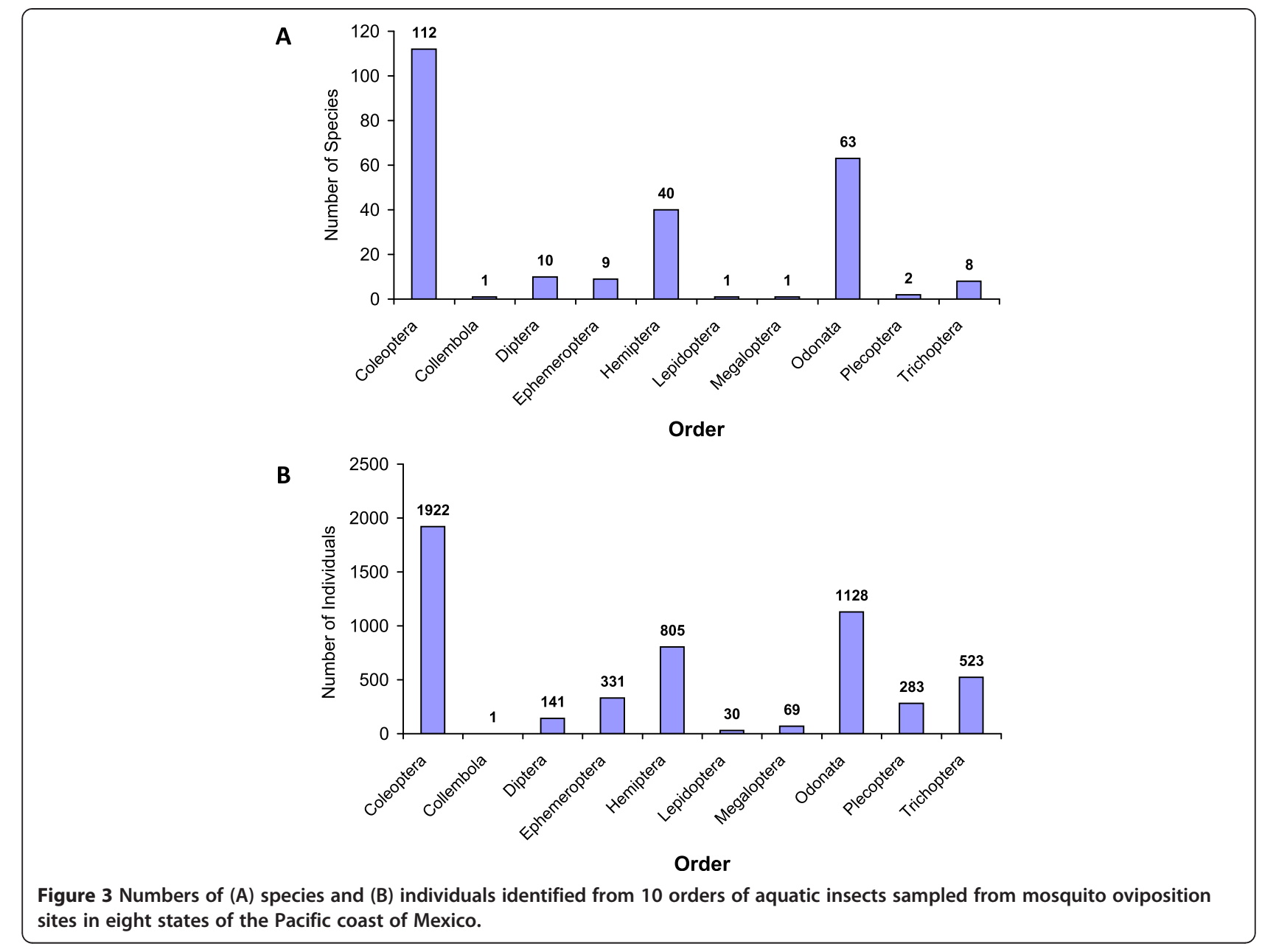

The selection of sampling sites within each state was based on established information available on the type of ecosystem and habitat used by anophelines and culicids as likely oviposition sites. Decisions on which habitats to sample, and in which period of the year, doubtless affected the likelihood of identifying particular species. This is because the oviposition site preferences of each mosquito species reflect a characteristic and habitatspecific suite of biotic and abiotic factors that favor the development and survival of their offspring [9]. However, such targeted sampling regimes are an inevitable consequence of finite financial and human resources available for faunistic studies of this kind.

Globally, the family Culicidae comprises 3,525 species distributed among 111 genera [49]. Mosquito diversity tends to be highest in tropical habitats [4]. In the present study a total of 15 genera and 74 species were identified, which represents $\sim 2 \%$ of global species. Within Mexico, the recorded mosquito fauna consists of between 15 and 16 genera and between 217 and 239 species [5,6], although the genera Chagasia, Orthopodomyia, Shannoniana, and Toxorhynchites were excluded from these reports because these genera have no species of medical importance. The mosquito species reported in the present study represented 30 - 34\% of the total Mexican mosquito fauna. Surveys on the mosquito fauna of Mexico are remarkably sparse. A total of 52 species from 11 genera have been recorded for the state of Guerrero State [17,28,29], in addition to species from other genera, such as Toxorhynchites species, that were not considered in the present study. This compared with 38 species from 10 genera recorded in the present study. In the remaining seven states 17-54 species were identified from 7-14 genera with the highest taxa richness in the state of Chiapas and the lowest in Nayarit. Overall, the present study extended the number of species records by just two species in Guerrero state and by up to 14 species in Chiapas state (Table 1). Similarly, the percentage of recorded species was increased by just $4 \%$ in Guerrero ( 2 additional species/52 previously reported species), but was almost doubled for Colima (9 new records/10 reported species), underlining the value of the present study to understanding the distribution of vector species in this region. Surveys have also been conducted in other states of Mexico, such as 


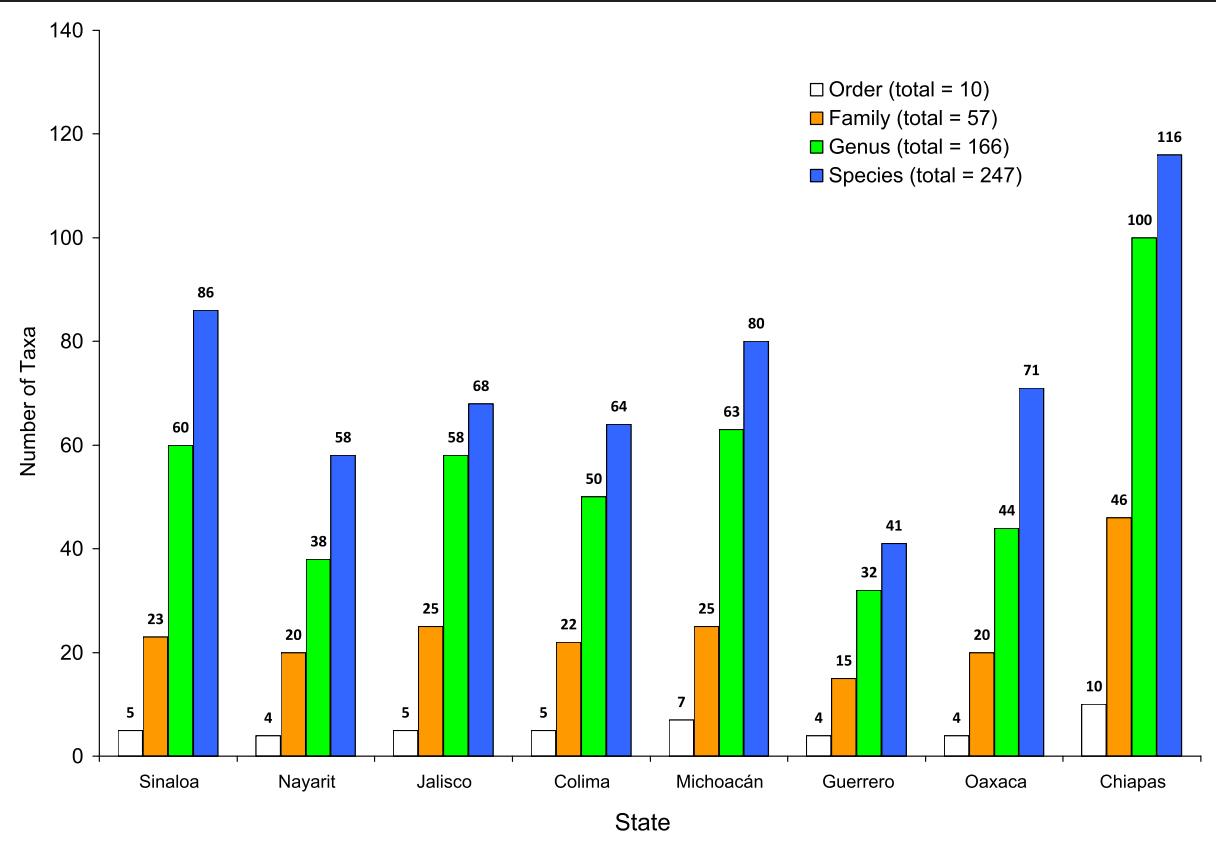

Figure 4 Taxa richness of aquatic insects sampled from mosquito oviposition sites in eight states of the Pacific coast of Mexico.

Veracruz [7], Yucatán [50-52], and Quintana Roo [53] but their geographical location, $\sim 1000 \mathrm{~km}$ from the Pacific coast of Mexico, and marked differences in the type of ecosystems present in the Atlantic and Caribbean coasts compared to the Pacific coast, means that these studies are of limited relevance to the present findings.

The diversity index values of mosquitoes in the eight states included in this study ranged from 1.93 for the state of Michoacan to 2.66 for the state of Chiapas. There are no previous systematic surveys of mosquitoes in the Pacific coast region, with the exception of the studies performed in the 1950's [28,29], and one study in Guerrero in the 1970's [17]. The high mosquito diversity present in the Pacific coast region is likely due to a combination of the overlap between Neartic and Neotropical fauna and the great diversity of ecosystems present in this region, which is largely responsible for the status of Mexico as one of the world's megadiverse countries. In this respect, the states of Chiapas and Oaxaca represent the states with the highest diversity in Mexico, followed by the states of Veracruz, Guerrero and Michoacán [54].

Globally, 465 species of Anopheles have been recognized, of which approximately 70 species have the capacity to transmit human malaria parasites [9,55], and 41 are considered to be dominant vector species, capable of transmitting malaria at a level of major concern to public health [56]. Approximately one third of the population of Mexico lives in areas prone to malaria transmission [57]. Between 26 and 28 species of Anopheles have been reported in Mexico [5,6,58], but only two of these are considered as being of major public health concern: $A n$. pseudopunctipennis and An. albimanus are the principal vectors of $P$. vivax in mountain foothills and coastal areas, respectively $[13,59]$.

A total of 18 species of anophelines were recorded during this study, in addition to one previous species record (Anopheles xelajuensis) [28]. The state of Chiapas had the highest anopheline species richness (15) and the highest diversity value (1.61), followed by the states of Michoacan (9 species) and Oaxaca (9 species). Our study expands the anopheline fauna of seven of the eight states studied (Table 1). Specifically, An. crucians is a new species record for Chiapas, Oaxaca and Colima states; $A n$. franciscanus is a new record for Chiapas, Oaxaca and Jalisco states; An. freeborni is a new record for Michoacán state; An. punctimacula is a new record for Sinaola state, and An. punctipennis is a new record for Jalisco and Nayarit states. No new anopheline records were obtained for the state of Guerrero [17].

The mosquitoes Ae. aegypti and Ae. albopictus are vectors of dengue in tropical and subtropical regions throughout the world and represent a major public health concern [60]. However, despite its dramatic global expansion in the last three decades, Ae. albopictus is believed to have a lesser role in dengue virus transmission compared to Ae. aegypti, due to differences in host preference and vector competence [61]. Of these, only Ae. aegypti was present in all eight states of our study area, whereas the exotic invasive species Ae. albopictus was only present in Chiapas. In Mexico, Ae. albopictus was first recorded in 1988 in the northeastern state of Tamaulipas [62,63], but was also reported in the 


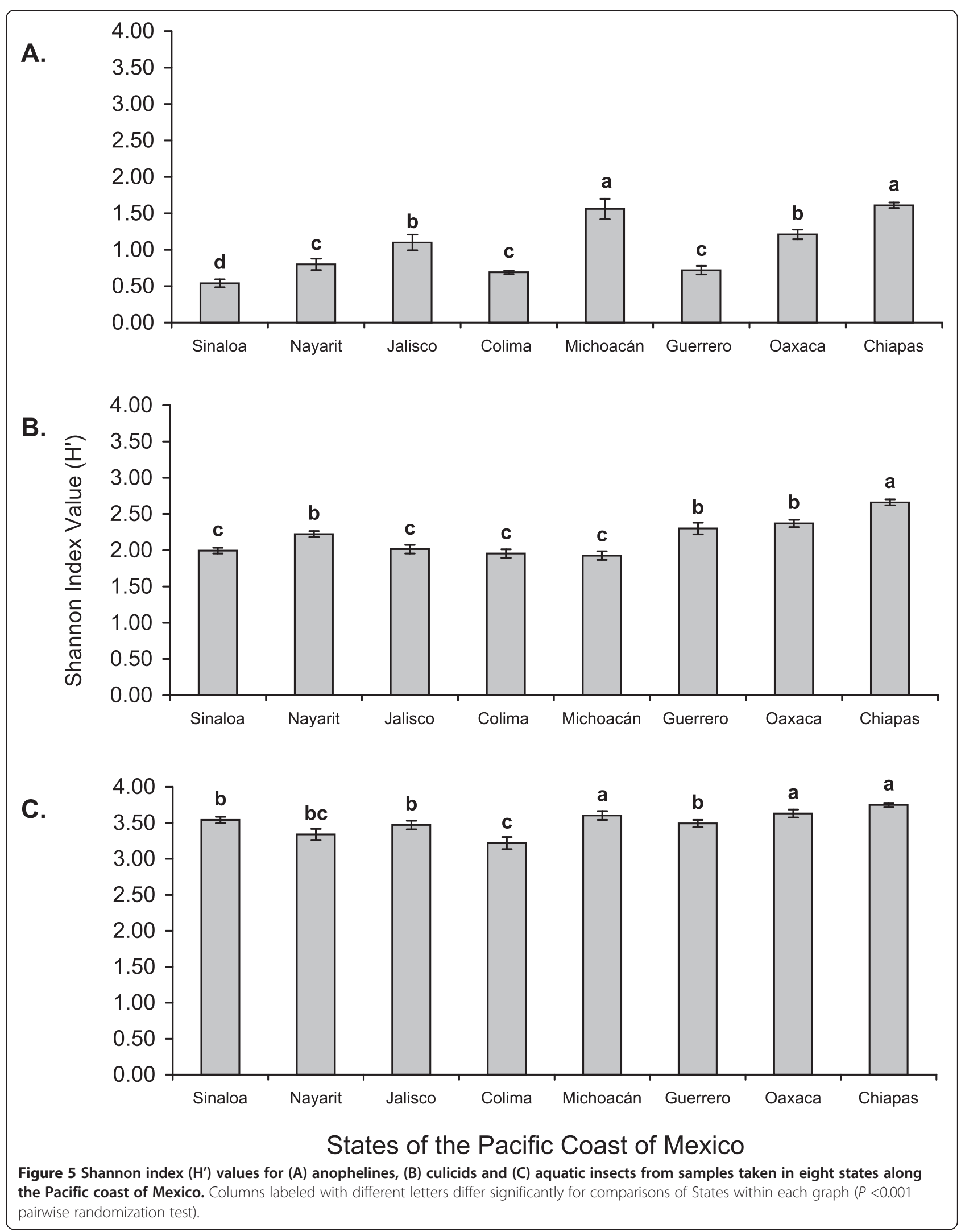


Table 3 Values of total diversity estimated by jackknife for culicid, anopheline and aquatic insects sampled in eight states of the Pacific coast of Mexico

\begin{tabular}{|c|c|c|c|c|c|c|}
\hline Source & $\begin{array}{c}\text { Number of analyzed } \\
\text { samples** }\end{array}$ & Values of $H^{\prime}$ & Pseudovalues $\varphi$ & Jackknifing $( \pm \mathrm{SE})$ & $\begin{array}{c}\text { Confidence } \\
\text { limits }(95 \%)^{*} \\
\end{array}$ & Error (\%) \\
\hline \multirow[t]{10}{*}{ Culicids } & All (8) & 2.87 & & & & \\
\hline & $(2,3,4,5,6,7,8)$ & 2.72 & 2.81 & & & \\
\hline & $(1,3,4,5,6,7,8)$ & 2.88 & 1.06 & & & \\
\hline & $(1,2,4,5,6,7,8)$ & 2.88 & 1.48 & & & \\
\hline & $(1,2,3,5,6,7,8)$ & 2.89 & 1.48 & $3.00 \pm 0.15$ & $2.65-3.35$ & 4.57 \\
\hline & $(1,2,3,4,6,7,8)$ & 2.88 & 1.48 & & & \\
\hline & $(1,2,3,4,5,7,8)$ & 2.84 & 1.69 & & & \\
\hline & $(1,2,3,4,5,6,8)$ & 2.82 & 1.27 & & & \\
\hline & $(1,2,3,4,5,6,7)$ & 2.90 & 1.06 & & & \\
\hline & All (8) & 1.48 & & & & \\
\hline \multirow[t]{8}{*}{ Anophelines } & $(2,3,4,5,6,7,8)$ & 1.29 & 3.92 & & & \\
\hline & $(1,3,4,5,6,7,8)$ & 1.54 & 2.80 & & & \\
\hline & $(1,2,4,5,6,7,8)$ & 1.48 & 2.80 & & & \\
\hline & $(1,2,3,5,6,7,8)$ & 1.48 & 2.73 & $1.54 \pm 0.20$ & $1.07-2.01$ & 4.14 \\
\hline & $(1,2,3,4,6,7,8)$ & 1.48 & 2.80 & & & \\
\hline & $(1,2,3,4,5,7,8)$ & 1.45 & 3.08 & & & \\
\hline & $(1,2,3,4,5,6,8)$ & 1.51 & 3.22 & & & \\
\hline & $(1,2,3,4,5,6,7)$ & 1.54 & 2.66 & & & \\
\hline \multirow[t]{9}{*}{ Aquatic insects } & All (8) & 4.46 & & & & \\
\hline & $(2,3,4,5,6,7,8)$ & 4.44 & 4.60 & & & \\
\hline & $(1,3,4,5,6,7,8)$ & 4.45 & 4.53 & & & \\
\hline & $(1,2,4,5,6,7,8)$ & 4.45 & 4.53 & & & \\
\hline & $(1,2,3,5,6,7,8)$ & 4.45 & 4.53 & $4.61 \pm 0.04$ & $4.52-4.70$ & 3.34 \\
\hline & $(1,2,3,4,6,7,8)$ & 4.42 & 4.74 & & & \\
\hline & $(1,2,3,4,5,7,8)$ & 4.46 & 4.46 & & & \\
\hline & $(1,2,3,4,5,6,8)$ & 4.41 & 4.81 & & & \\
\hline & $(1,2,3,4,5,6,7)$ & 4.43 & 4.67 & & & \\
\hline
\end{tabular}

*Confidence intervals were calculated by bootstrap.

**The numbers 1 to 8 represent each of the states in the following order, 1. Chiapas, 2. Guerrero, 3. Oaxaca, 4. Colima, 5. Jalisco, 6. Michoacán, 7. Nayarit, 8. Sinaloa.

southern state of Chiapas in 2002 [64], and recently in the central state of Morelos [65] and the Gulf coast states of Yucatán [66], and Veracruz [67]. Given the presence of Ae. albopictus in Chiapas, its introduction, establishment, and spread to the other states of the Pacific coast is an issue of considerable concern. As invasive mosquitoes have the potential for biotic impacts on native species, ecosystems, and on human and animal health [68], an invasive mosquito that replaces a resident species via competition may alter disease transmission and amplify the importance of vector borne disease in affected areas [69].

The taxa richness and diversity of aquatic insects associated with breeding sites of mosquitoes in this study was high, with a total of 10 orders, 57 families, 166 genera and 247 species, with Shannon index values between 3.22 and 3.75, depending on the state in question. The orders of greatest abundance and species richness were Coleoptera, Odonata and Hemiptera that represented $87 \%$ of the total species sampled.

In northern Mexico, 39 genera, 27 families, and 7 orders of aquatic insects were reported in association with oviposition sites of An. pseudopunctipennis [18]. Similarly, 52 genera, 19 families, and 3 orders of aquatic insects were reported in association with An. albimanus oviposition sites in southern Chiapas, of which Coleoptera was the most abundant and diverse order [19]. In comparison, another study in southern Chiapas reported 90 genera, 40 families and 10 orders of aquatic insects associated with oviposition sites of An. pseudopunctipennis. In 


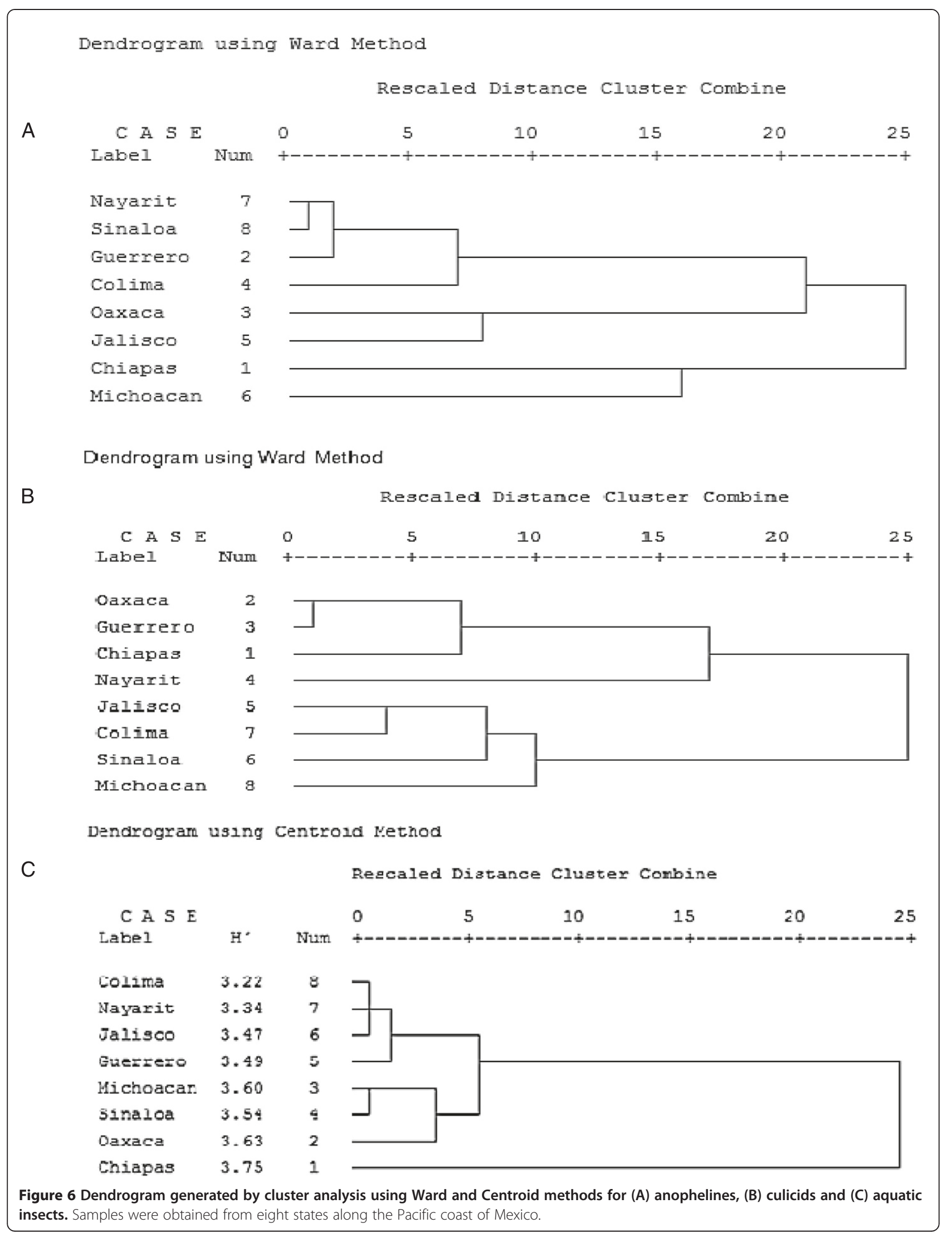


Table 4 Similarity values for species composition of mosquitoes from eight states of the Pacific coast of Mexico, using Morisita Horn index

\begin{tabular}{ccccccccc}
\hline & Chiapas & Oaxaca & Guerrero & Nayarit & Jalisco & Sinaloa & Colima & Michoacán \\
\hline Chiapas & 1.00 & 0.71 & 0.73 & 0.51 & 0.27 & 0.44 & 0.43 & 0.20 \\
Oaxaca & 0.71 & 1.00 & 0.91 & 0.53 & 0.48 & 0.52 & 0.50 & 0.32 \\
Guerrero & 0.73 & 0.91 & 1.00 & 0.60 & 0.43 & 0.55 & 0.56 & 0.27 \\
Nayarit & 0.51 & 0.53 & 0.60 & 1.00 & 0.26 & 0.51 & 0.27 & 0.16 \\
Jalisco & 0.27 & 0.48 & 0.43 & 0.26 & 1.00 & 0.46 & 0.68 & 0.91 \\
Sinaloa & 0.44 & 0.52 & 0.55 & 0.51 & 0.46 & 1.00 & 0.69 & 0.36 \\
Colima & 0.43 & 0.50 & 0.56 & 0.27 & 0.68 & 0.69 & 1.00 & 0.57 \\
Michoacán & 0.20 & 0.32 & 0.27 & 0.16 & 0.91 & 0.36 & 0.57 & 1.00 \\
\hline
\end{tabular}

this case Shannon index values fluctuated between 2.4 and 3.2 [21]. However, these previous studies were focused on the oviposition sites of single species of mosquitoes, and restricted to a single state, which contrasts with our effort to evaluate the diversity of aquatic insects associated with immature mosquitoes along the Pacific coast of Mexico. Comparable studies in temperate regions have reported markedly lower diversity of aquatic insects, which was ascribed to the harsh environmental conditions and instability of the habitat studied [11].

The presence of mosquito larvae was not significantly correlated with the abundance of aquatic insect predators of the orders Coleoptera and Hemiptera. A negative tendency was observed in the correlation between mosquito abundance and predator abundance, but in neither case was this significant, probably due to the large number of observations of single individuals in the dataset used. Previous studies have highlighted the importance of these orders, since many members of these orders are known to prey on mosquito larvae [26,70]. In addition to regulating mosquito populations by direct predation, a number of these predators can influence adult mosquito oviposition decisions or affect the rate of development of immature stages [10,71-73]. There is renewed interest in employing natural enemies for the control of mosquito larvae to complement existing vector control measures. In this respect, due to their numerical and functional responses, naturally occurring predators can be a significant density dependent mortality factor in the regulation of mosquito populations $[74,75]$. However, apart from some species of fish and bacteria-based biological insecticides, the adoption of biological vector control in most countries remains extremely limited.

\section{Conclusion}

This study represents the first systematic update to the inventory and distribution of mosquitoes in Mexico in over five decades. The majority of the individuals reported were catalogued in databases of mosquitoes and aquatic insects in Mexico's National Biodiversity Information System
(SNIB-CONABIO), and are available for public inspection. We believe this represents a valuable contribution to recording the diversity and geographic distribution of the mosquitoes and aquatic insects in this region that is affected by major vector borne diseases, particularly dengue and malaria. Numerous new species records for different states along the Pacific coast are reported. Considerably greater sampling effort would be required to yield realistic estimates of total mosquito species richness of the country, particularly for the Mexican Culicidae inventory, given the great diversity of ecosystems present in this megadiverse country.

\section{Additional file}

Additional file 1: Table S1. Numbers of field-collected mosquitoes that were reared in the laboratory and identified to species following adult emergence. Columns indicate numbers of each species in samples taken from eight states of the Pacific coast of Mexico.

\section{Competing interests}

The authors declare that they have no competing interests.

\section{Authors' contributions}

MCM obtained funding via a competitive proposal. MCM, JGB, CFM, AU, AOB, MM performed field studies. JGB, MCM, CFM, AU, MM performed mosquito rearing. $A O B$ identified mosquitoes. JGB, HQM, RNG identified aquatic insects. JGB, TW performed statistical analyses. JGB, CFM, TW wrote the manuscript. All authors read and approved the final version of the manuscript.

\section{Acknowledgement}

We thank José Luis Aguilar Rodríguez, Martín Vázquez Castillo and José Luis Espinosa Aguilar for technical assistance.

\section{Financial support}

The study was funded by CONABIO projects V019 and FE009.

\section{Author details}

${ }^{1}$ Centro Regional de Investigación en Salud Pública - INSP, Tapachula, Chiapas, Mexico. ${ }^{2}$ Facultad de Ciencias Biológicas, Universidad Autónoma de Nuevo León, Nuevo León, Mexico. ${ }^{3}$ Instituto de Ecología AC, Xalapa, Veracruz, Mexico. 


\section{References}

1. Campbell-Lendrum D, Molyneux D, Amerasinghe F: Ecosystems and vector-borne disease control. In Ecosystems and Human Well-Being. Findings of the responses working group of the Millennium Ecosystem Assessment. Volume 3. Edited by Epstein P, Githeko A, Rabinovich J, Weinstein P. Washington DC: Island Press; 2005:353-372.

2. Townson H, Nathan MB, Zaim M, Guillet P, Manga L, Bos R, Kindhauser M: Exploiting the potential of vector control for disease prevention. Bull WHO 2005, 83:942-947.

3. WHO: International Statistical Classification of Diseases and Related Health Problems 10th Revision (ICD-10). 2010. http://apps.who.int/classifications/ icd10/browse/2010/en\#/B74.

4. Harbach RE: The Culicidae (Diptera): A review of taxonomy, classification and phylogeny. Zootaxa 2007, 1668:591-638.

5. Darsie RF Jr: A survey and bibliography of the mosquito fauna of Mexico (Diptera: Culicidae). J Am Mosa Contr Assoc 1996, 12:298-306.

6. Ibáñez-Bernal S, Strickman S, Martínez-Campos C: Culicidae (Diptera). In Biodiversidad, Taxonomía y Biogeografía de Artrópodos de México: Hacia una Síntesis de Su conocimiento. Edited by Llorente BJ, García AN, González E. Mexico City: Universidad Nacional Autónoma de México; 1996:591-602.

7. Beltrán-Aguilar A, Ibáñez-Bernal S, Mendoza-Palmero F, Sandoval-Ruiz CA Hernández-Xoliot RA: Taxonomía y distribución de los anofelinos en el Estado de Veracruz, México (Diptera: Culicidae, Anophelinae). Acta Zool Mex 2011, 27:601-755.

8. Knight KL, Stone A: A Catalog of the Mosquitoes of the World (Diptera: Culicidae). Volume 6. The Thomas Say Foundation, Langham, Maryland: Entomological Society of America; 1977.

9. Service MW: Medical Entomology for Students. 3rd edition. Cambridge, UK: Cambridge University Press; 2004.

10. Juliano SA: Species interactions among larval mosquitoes: context dependence across habitat gradients. Ann Rev Entomol 2009, 54:37-56.

11. Rochlin I, Dempsey ME, Iwanejko T, Ninivaggi DV: Aquatic insects of New York salt marsh associated with mosquito larval habitat and their potential utility as bioindicators. J Ins Sci 2011, 11:172

12. Savage HM, Rejmankova E, Arredondo-Jiménez Jl, Roberts DR, Rodríguez MH: Limnological and botanical characterization of larval habitats for two primary malarial vectors, Anopheles pseudopunctipennis and Anopheles albimanus, in coastal areas of Chiapas State, Mexico. J Am Mosa Contr Assoc 1990, 6:612-620.

13. Fernández-Salas I, Rodríguez MH, Roberts DR, Rodríguez MC, Wirtz RA: Bionomics of adult Anopheles pseudopunctipennis (Diptera: Culicidae) in the Tapachula foothills area of Southern Mexico. J Med Entomol 1994, 3:663-670.

14. Manguin S, Roberts DR, Peyton EL, Rejmankova E, Pecor J: Characterization of Anopheles pseudopunctipennis larval habitats. J Am Mosa Contr Assoc 1996, 12:619-626.

15. Rubio-Palis Y, Zimmerman RH: Ecoregional classification of malaria vectors in the neotropics. J Med Entomol 1997, 34:499-510.

16. Sinka ME, Rubio-Palis Y, Manguin S, Patil AP, Temperley WH, Boeckel TV, Kabaria CW, Harbach RE, Hay S, Gething PW: The dominant Anopheles vectors of human malaria in the Americas: occurrence data, distribution maps and bionomic précis. Parasit Vectors 2010, 3:117.

17. García-Aldrete AN, Pletsch DJ: Fauna de mosquitos (Diptera: Culicidae), en la zona turística de Ixtapa, Guerrero. Folia Entomol Mex 1976, 34:71-83.

18. Delgado-Gallardo ML, Badii MH, Quiroz-Martinez H: Diversidad ecológica de las comunidades acuáticas cohabitando con Anopheles pseudopunctipennis (Diptera: Culicidae) en el arroyo la Ciudadela, en el municipio de Benito Juárez, Nuevo León, México. Southwest Entomol 1994, 19:77-81.

19. Danis-Lozano R, Rodríguez MH, Arredondo-Jiménez Jl, Hernández-Ávila M, Mallorca C: Aquatic insects associated with Anopheles albimanus (Diptera: Culicidae) breeding sites in southern Mexico. Env Entomol 1997, 26:826-838.

20. Bond JG, Novelo-Gutiérrez R, Ulloa A, Rojas JC, Quiroz-Martínez H, Williams T: Diversity, abundance, and disturbance response of Odonata associated with breeding sites of Anopheles pseudopunctipennis (Diptera: Culicidae) in southern Mexico. Env Entomol 2006, 35:1561-1568.

21. Bond JG, Quiroz-Martinez H, Rojas JC, Valle J, Ulloa A, Williams T: Impact of environmental manipulation for Anopheles pseudopunctipennis Theobald control on aquatic insect communities in southern Mexico. J Vect Ecol 2007, 32:41-53.

22. Merritt $R$, Cummins K, Berg M: An introduction to the aquatic insects of North America. 4th edition. Dubuque, lowa: Kendall Hunt Publishing Co; 2008.
23. Ward JV: Aquatic Insect Ecology. Volume 1. Biology and Habitat. New York: John Wiley \& Sons; 1992.

24. Malmqvist B: Aquatic invertebrates in riverine landscapes. Freshwat Biol 2002, 47:679-694.

25. Rosenberg DM, Resh RH: Freshwater Biomonitoring and Benthic Macroinvertebrates. New York: Chapman \& Hall; 1993.

26. Shaalan EA, Canyon DV: Aquatic insect predators and mosquito control. Trop Biomed 2009, 26:223-261.

27. Instituto Nacional de Estadística Geografía e Informática: Anuario Estadístico de los Estados Unidos Mexicanos. Mexico City: Instituto Nacional de Estadística Geografía e Informática; 2000.

28. Vargas L, Martínez-Palacios A: Anofelinos Mexicanos, Taxonomía y Distribución. Mexico City: Comisión Nacional para la Erradicación del Paludismo: Secretaría de Salubridad y Asistencia; 1956.

29. Vargas L: Especies y distribución de mosquitos mexicanos no anofelinos. Rev Inst Salubr Enferm Trop 1956, 16:19-36.

30. Comité Nacional para la Vigilancia Epidemiológica: Aviso epidemiológico Virus del Oeste del Nilo: Incremento de casos de infección por Virus del Oeste del Nilo en los Estados Unidos de América. 2012. CoNaVe/05/NIRUS DEL OESTE DEL NILO. http://www.facmed.unam.mx/deptos/salud/ vigilanciaepidem/alerta_20ago2012.pdf.

31. Darsie RF Jr, Ward RA: Identification and Geographical Distribution of the Mosquitoes of North America, North of Mexico. 2nd edition. Gainesville: University of Florida; 2005.

32. Clark-Gill S, Darsie RF Jr: The mosquitoes of Guatemala, their identification, distribution and bionomics, with keys to adult females and larvae. Mosa Systemat 1983, 15:151-284.

33. Wilkerson RC, Strickman D, Fernández-Salas I, Ibáñez-Bernal S, Liwak TR: Clave ilustrada para la identificación de las hembras de mosquitos anofelinos de México y América Central. Mexico City: Secretaría de Salud. Centro de Investigación de Paludismo; 1993.

34. Pollard JE: Investigator differences associated with a kicking method for sampling macroinvertebrates. J Freshwat Ecol 1981, 1:215-224.

35. González-Soriano E, Novelo-Gutiérrez R: Odonata. In Biodiversidad, taxonomía y biogeografía de artrópodos de México: Hacia una síntesis de su conocimiento. Edited by Llorente BJ, García AAN, González SE. Mexico City: Universidad Autónoma de México; 1996:147-167.

36. Westfall MJ Jr, May ML: Damselflies of North America. Gainesville: Scientific Publishers; 1996

37. Novelo-Gutiérrez R: Clave para la separación de familias y géneros de las náyades de Odonata de México. Parte I. Zygoptera. Dugesiana 1997, 4:1-10

38. Novelo-Gutiérrez R: Clave para la identificación de familias y géneros de las náyades de Odonata de México. Parte II. Anisoptera. Dugesiana 1997, 4:31-40.

39. Contreras-Ramos A: List of species of Neotropical Megaloptera (Neuropterida). Proc Entomol Soc Wash 1999, 101:273-284.

40. Needham JG, Westfall MJL, May M: Dragonflies of North America. Gainesville: Scientific Publishers; 2000.

41. Epler JH: Identification Manual for the Aquatic and Semi-aquatic Heteroptero of Florida (Belostomatidae, Corixidae, Gelastocoridae, Gerridae, Hebridae, Hydrometridae, Mesoveliidae, Naucoridae, Nepidae, Notonectidae, Ochteridae, Pleidae, Saldidae, Veliidae). 2006. http://bugguide.net/node/view/368765.

42. Epler JH: The Water Beetles of Florida - an Identification Manual for the families Chrysomelidae, Curculionidae, Dryopidae, Dytiscidae, Elmidae, Gyrinidae, Haliplidae, Helophoridae, Hydraenidae, Hydrochidae, Hydrophilidae, Noteridae, Psephenidae, Ptilodactylidae and Scirtidae. 2010. http://publicfiles.dep.state.fl. us/dear/labs/biology/biokeys/beetles10.pdf.

43. Comisión Nacional para el Conocimiento y Uso de la Biodiversidad (CONABIO): Sistema de Información Biótica 4.5. Manual de Usuario. Mexico City: Fideicomiso Fondo para la Biodiversidad; 2006.

44. Magurran AE: Measuring Biological Diversity. Oxford, UK: Blackwell; 2004

45. Henderson PA, Seaby RM: Species Diversity and Richness III v 3.0.2. Lymington, UK: Pisces Conservation Ltd; 2002.

46. Solow AR: A simple test for change in community structure. J Anim Ecol 1993, 62:191-193.

47. Southwood TRE, Henderson PA: Ecological Methods. 3rd edition. Oxford, UK: Blackwell; 2000.

48. Wolda H: Similarity indices, sample size and diversity. Oecologia 1981, 50:296-302

49. Harbach RE: Culicidae (Diptera). Mosquito Taxonomic Inventory. 2013. http://mosquito-taxonomic-inventory.info/valid-species-list. 
50. Nájera-Vázquez R, Dzul F, Sabido M, Tun-Ku E, Manrique-Saide P: New distribution records of mosquitoes (Diptera: Culicidae) for Yucatan, Mexico. Entomol News 2004, 115:181-190.

51. Zapata-Peniche A, Manrique-Saide P, Rebollar-Téllez EA, Che-Mendoza A Dzul-Manzanilla F: Identificación de larvas de mosquitos (Diptera: Culicidae) de Mérida, Yucatán, México y sus principales criaderos. Rev Biomed 2007, 18:3-17.

52. Dzul MF, Manrique SP, Che MA, Rebollar TE: Mosquitos de Yucatán. In Biodiversidad y Desarrollo Humano en Yucatán. Edited by Durán R, Méndez M. Mérdida, México: CICY, PPD-FMAM, CONABIO, SEDUMA; 2010. http://www.seduma.yucatan.gob.mx/biodiversidad-yucatan/libro-biodiversidadyucatan.php.

53. Ortega MAl, Avila PM, Elizondo-Quiroga A, Harbach RE, Quetzaly K, SillerRodríguez QK, Fernández-Salas I: The mosquitoes of Quintana Roo State, Mexico (Diptera: Culicidae). Acta Zool Mex 2010, 26:33-46.

54. Mittermeier RA, Goesttsch C: La importancia de la diversidad biológica de México. In México ante los retos de la biodiversidad. Edited by Sarukhán J, Dirzo R. Mexico: Mexico City: CONABIO; 1992.

55. Service MW, Townson H: The Anopheles vector. In Essential Malariology. 4th edition. Edited by Gilles HM, Warrell DA. London: Arnold; 2002:59-84.

56. Sinka ME, Bangs MJ, Manguin S, Rubio-Palis Y, Chareonviriyaphap T, Coetzee M, Mbogo CM, Hemingway J, Patil AP, Temperley WH, Gething PW, Kabaria CW, Burkot TR, Harbach RE, Hay SI: A global map of dominant malaria vectors. Parasit Vectors 2012, 5:69.

57. Rodríguez MH, Hernández-Ávila JE, Betanzos-Reyes AF, Danis-Lozano R, González-Cerón L, Durán-Arenas LG, Méndez-Galván JF, Vázquez-Mellado RM, Velásquez-Monroy OJ, Holguín-Bernal, Tapia-Coyner R: An ecosystem approach study of malaria transmission and control interventions in southern Mexico. Forum 8. Mexico City: Global Forum for Health Research; 2004. http://bvs.per.paho.org/texcom/cd048449/rodrigue.pdf.

58. Gaffigan TV, Wilkerson RC, Pecor JE, Stoffer JA, Anderson T: Systematic Catalog of Culicidae. Silver Spring, Maryland: Walter Reed Army Institute of Research. Walter Reed Biosystematics Unit: Division of Entomology. http://www.mosquitocatalog.org/default.aspx?pglD=2.

59. Rodriguez MH, Loyola EG: Malaria in Mexico. In Proceedings and Papers of the 58th Annual Conference of the California Mosquito and Vector Control Association, 28-31 January 1990. Edited by Sparks NV. Sacramento, CA: CMVCA Press; 1990:49-52.

60. Brady OJ, Johansson MA, Guerra CA, Bhatt S, Golding N, Pigott DM, Delatte H, Grech MG, Leisnham PT, Maciel-de-Freitas R, Styer LM, Smith DL, Scott TW, Gething PW, Hay SI: Modelling adult Aedes aegypti and Aedes albopictus survival at different temperatures in laboratory and field settings. Parasit Vectors 2013, 6:351.

61. Lambrechts L, Scott TW, Gubler DJ: Consequences of the expanding global distribution of Aedes albopictus for dengue virus transmission. PLoS Negl Trop Dis 2010, 4:1-10.

62. Womack ML: Distribution, abundance and bionomics of Aedes albopictus in southern Texas. J Am Mosa Contr Assoc 1993, 9:367-369.

63. Ibáñez-Bernal S, Martínez-Campos C: Aedes albopictus in Mexico. J Am Mosq Contr Assoc 1994, 10:231-232.

64. Casas-Martínez M, Torres-Estrada JL: First evidence of Aedes albopictus (Skute) in Southern Chiapas, Mexico. Emerg Infect Dis 2003, 9:606-607.

65. Villegas-Trejo A, Manrique-Saide P, Che-Mendoza A, Cruz-Canto W, Fernandez MG, González-Acosta C, Dzul-Manzanilla F, Huerta H, Arredondo-Jiménez Jl: First report of Aedes albopictus and other mosquito species in Morelos, Mexico. J Am Mosq Contr Assoc 2010, 26:321-323.

66. Salomón-Grajales J, Lugo-Moguel GV, Tinal-Gordillo VR, de la Cruz-Velázquez J, Beaty BJ, Eisen L, Lozano-Fuentes S, Moore CG, García-Rejón JE: Aedes albopictus mosquitoes, Yucatan peninsula, Mexico. Emerg Infect Dis 2012, 18:525-527.

67. de la Cruz-Francisco V, Veda-Moreno DI, Valdés-Murillo A: Ecological aspects of larval incidence of mosquitoes (Diptera: Culicidae) in Tuxpan, Veracruz, Mexico. Rev Colomb Entomol 2012, 38:128-133.

68. Lounibos LP: Invasions by insect vectors of human disease. Annu Rev Entomol 2002, 47:233-266.

69. Juliano SA, Lounibos LP: Ecology of invasive mosquitoes: effects on resident species and on human health. Ecol Lett 2005, 8:558-574.

70. Culler LE, Lamp WO: Selective predation by larval Agabus (Coleoptera: Dytiscidae) on mosquitoes: support for conservation based mosquito suppression in constructed wetlands. Freshwat Biol 2009, 54:2003-2014.

71. Kumar R, Hwang J: Larvicidal efficiency of aquatic predators: a perspective for mosquito biocontrol. Zool Stud 2006, 45:447-466.
72. Legner EF: Biological control of Diptera of medical and veterinary importance. J Vect Ecol 1994, 20:59-120.

73. Knight TM, Chase JM, Goss JM, Knight JJ: Effects of interspecific competition, predation, and their interaction on survival and development time of immature Anopheles quadrimaculatus. J Vect Ecol 2004, 29:277-284.

74. Kweka EJ, Zhou G, Gilbreath TM III, Afrane Y, Nyindo M, Githeko AK, Yan G: Predation efficiency of Anopheles gambiae larvae by aquatic predators in western Kenya highlands. Parasit Vectors 2011, 4:128.

75. Mereta ST, Yewhalaw D, Boets P, Ahmed A, Duchateau L, Speybroeck N, Vanwambeke SO, Legesse W, De Meester L, Goethals PLM: Physicochemical and biological characterization of anopheline mosquito larval habitats (Diptera: Culicidae): implications for malaria control. Parasit Vectors 2013, 6:320.

doi:10.1186/1756-3305-7-41

Cite this article as: Bond et al:: Diversity of mosquitoes and the aquatic insects associated with their oviposition sites along the Pacific coast of Mexico. Parasites \& Vectors 2014 7:41.

\section{Submit your next manuscript to BioMed Central and take full advantage of:}

- Convenient online submission

- Thorough peer review

- No space constraints or color figure charges

- Immediate publication on acceptance

- Inclusion in PubMed, CAS, Scopus and Google Scholar

- Research which is freely available for redistribution 